\title{
Magnetic resonance imaging of the elbow
}

\author{
Alecio Lombardi ${ }^{1,2, A, B, E, F}$, Aria Ashir ${ }^{2,3, B, E, F}$, Tetyana Gorbachova ${ }^{4, A, B, E}$, Mihra S. Taljanovic ${ }^{5,6, A, A, B, E, F}$, Eric Y. Chang ${ }^{1,2, A, B, E}$ \\ 'Research Service, VA San Diego Healthcare System, San Diego, CA, USA \\ ${ }^{2}$ Department of Radiology, University of California, San Diego, CA, USA \\ ${ }^{3}$ Drexel University College of Medicine, Philadelphia, PA, USA \\ ${ }^{4}$ Department of Radiology, Einstein Healthcare Network, Philadelphia, PA, USA \\ ${ }^{5}$ Department of Medical Imaging, University of Arizona/Banner University Medical Center, Tucson, AZ, USA \\ ${ }^{6}$ Simon Med Imaging, Scottsdale, AZ, USA
}

\section{Abstract}

\begin{abstract}
Elbow pain can cause disability, especially in athletes, and is a common clinical complaint for both the general practitioner and the orthopaedic surgeon. Magnetic resonance imaging (MRI) is an excellent tool for the evaluation of joint pathology due to its high sensitivity as a result of high contrast resolution for soft tissues. This article aims to describe the normal imaging anatomy and biomechanics of the elbow, the most commonly used MRI protocols and techniques, and common MRI findings related to tendinopathy, ligamentous and osteochondral injuries, and instability of the elbow.
\end{abstract}

Key words: MRI, elbow, epicondylosis, biceps brachii, cubital tunnel syndrome, radial tunnel syndrome.

\section{Introduction}

It is essential to understand the anatomy and function of the elbow in order to recognise common pathological imaging findings and correlate them with clinical manifestations. The understanding and proper implementation of the magnetic resonance imaging (MRI) protocol is also crucial for the correct interpretation of these findings. Finally, knowing the mechanisms of the most common elbow injuries and how they can affect joint function will facilitate communication between the radiologist and referring physician. Therefore, this article intends to review the use of MRI in the clinical evaluation of the elbow.

\section{Anatomy and biomechanics}

The elbow is a complex joint consisting of three articulations: the humeroradial, humeroulnar, and proximal radioulnar. The humeroulnar is a hinge joint that allows flexion and extension movements, while the radioulnar joint is a trochoid joint, which enables pivot motion translated in supination and pronation. The elbow can flex and extend between 0 and $140^{\circ}$ and can rotate about $80^{\circ}$, although for the typical daily tasks only 30 to $130^{\circ}$ of flexion and extension, and $50^{\circ}$ of rotation are necessary $[1,2]$. Flexion-extension occurs at the humeral trochlea, and supination-pronation occurs mostly along the capitellum and the radial head, with some contribution of the distal radioulnar joint [3]. The elbow has a slight normal valgus position between the humeral and ulnar shafts in extension called the carrying angle. This angle is higher in males compared to females and higher in adults compared to children $[4,5]$. An increased carrying angle may be associated with ulnar neuropathy [6].

The trochlea and the capitellum present mild internal rotation, valgus, and anterior rotation in relation to the long axis of the humerus. The capitellum is round and occupies a small portion of the distal humerus, while the trochlea is more extensive with a central depression that may present different angles among individuals [7]. The coronoid and olecranon fossa are located anteriorly and posteriorly in the distal humerus, serving as recesses for the coronoid process and olecranon of the ulna, respectively, during flexion and extension of the elbow. There is a difference in the extent of cartilage coverage

\section{Correspondence address:}

Dr. Eric Y. Chang, Research Service, VA San Diego Healthcare System, San Diego, CA, USA, e-mail: ericchangmd@gmail.com

Authors' contribution:

A Study design · B Data collection · C Statistical analysis · D Data interpretation - E Manuscript preparation · F Literature search · G Funds collection 
between the trochlea, which has around $330^{\circ}$ of cartilage coverage, and the capitellum, which has only $180^{\circ}$ of cartilage coverage and ends abruptly in its posterior contour. This particular anatomy was previously called the pseudo defect of capitellum and should not be interpreted as an osteochondral defect $[8,9]$.

The proximal ulna has a mild posterior rotation that complements the anterior rotation of the humeral trochlea. The radial head and neck, on the other hand, present a slight internal angulation to its long axis, which also suits the valgus and internal rotation of the distal humerus [1]. The anterolateral portion of the radial head and the centre of the articular surface of the ulna (sigmoid fossa) are devoid of articular cartilage, which may be a reason why fractures commonly occur at these sites.

Elbow stability is maintained primarily by the humeroulnar joint and the anterior bundle of the ulnar collateral ligament (UCL), with a small contribution from the lateral ulnar collateral ligament (LUCL). The common flexor-pronator and extensor tendons, together with the humeroradial joint, are considered secondary stabilisers $[1,10,11]$.

Regarding joint movement, the primary elbow flexors are the brachialis, biceps, and brachioradialis muscles, whereas the triceps muscle is the primary extensor. As for the supination and pronation, the biceps and the pronator teres are the leading muscles, respectively.

\section{Ulnar collateral ligament complex}

The elbow joint is enveloped by a single capsule and has two ligament complexes at the medial and lateral sides. The medial UCL, also known as the UCL, has three bands or bundles: the anterior, posterior, and transverse (Figure 1A). The anterior bundle can be further subdivided into anterior and posterior bands $[12,13]$ or superficial and deep capsular portions [14]. The anterior bundle serves as the main restraint to valgus overload to the elbow, which is particularly important in athletes involved in overhead activities and weightlifting. The anterior bundle originates from the inferior portion of the medial epicondyle and inserts distally onto the medial aspect of the coronoid process (sublime tubercle of the ulna) or along a ridge of bone distal to the sublime tubercle. The exact distal insertion of the anterior bundle may vary among individuals $[15,16]$. It is better visualised in the coronal oblique plane on proton density and T1-weighted MRI as a low-signal-intensity structure with a broad origin interspersed by fat under the medial epicondyle and a thin distal insertion. There is improved sensitivity in its assessment on MR arthrography compared to conventional MRI $[17,18]$.

The posterior bundle of the UCL arises from the posteroinferior aspect of the medial epicondyle, inserting distally onto the posteromedial margin of the trochlear notch forming the floor of the cubital tunnel. It is better visualised on axial proton density (PD) and T1-weighted MR images. The posterior bundle has increased importance in valgus stabilisation of the elbow in higher degrees of flexion because of its origin slightly posterior to the centre of the movement, making it taut from $60^{\circ}$ to full flexion. The anterior bundle origin is in the centre of the flexion-extension movement, which makes it taut from $60^{\circ}$ to full extension, acting as the main stabiliser in that range of motion [2]. Surgical reconstruction of the posterior bundle of the UCL demonstrated good outcome in
A

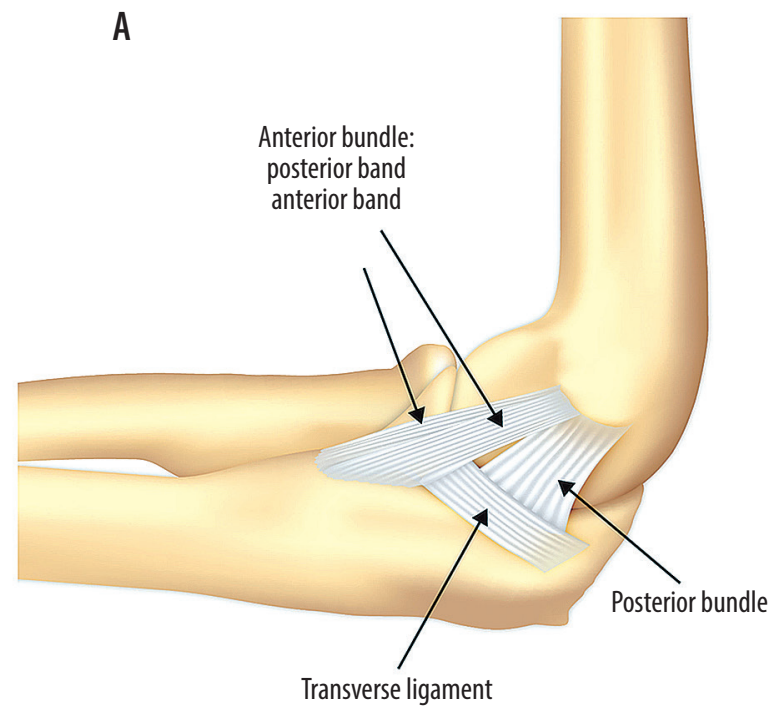

B

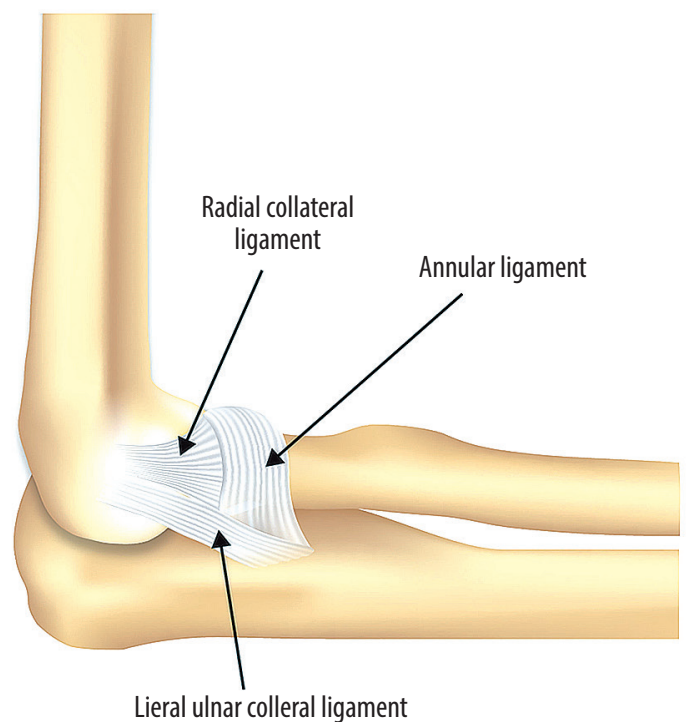

Figure 1. Schematics of the medial and lateral ligamentous complexes about the elbow. A) The ulnar collateral ligament complex is composed of the anterior, posterior, and transverse bundles. The anterior bundle is further subdivided into anterior and posterior bands. B) The lateral collateral ligament is composed of the radial collateral ligament, the lateral ulnar collateral ligament, and the annular ligament 
valgus extension overload (VEO) syndrome and varus posteromedial rotatory instability of the elbow (VPMRI), which will be discussed later in this article [19,20].

The thin transverse bundle of the UCL connects the distal attachments of the anterior and posterior bundles, does not have an important biomechanical role, and is not routinely characterised on MRI.

\section{Lateral collateral ligament complex}

The lateral collateral ligament complex (LCL), also known as the radial collateral ligament complex, has four elements: the LUCL, the radial collateral ligament (RCL), the annular ligament, and the accessory lateral collateral ligament (Figure 1B). The LUCL and radial collateral ligaments have a common origin from the lateral humeral epicondyle. The LUCL courses posterior to the radial head and inserts into the lateral aspect of the ulna (supinator crest) being the main stabiliser of the lateral elbow during varus stress. The RCL runs slightly anterior to the LUCL and blends distally with the annular ligament and supinator muscle [21]. Both are better visualised on coronal oblique T1- or PD-weighted MRI as thin, elongated, low signal intensity structures. The RCL is located anteriorly and sometimes can be visible in a single slice, whereas the LUCL is located posteriorly and, because of its oblique longitudinal course, is typically visualised in two or three slices. The annular ligament inserts on the anterior and posterior portions of the radial facet of the ulna (the lesser sigmoid notch), encircling the radial head, and serves as the primary stabiliser of the proximal radioulnar joint during supination and pronation $[22,23]$. It is better visualised on axial PD- or T1-weighted MRI, which is also useful to depict the distal insertion of the LUCL. Finally, the accessory lateral collateral ligament originates from the annular ligament and inserts into the supinator crest, stabilising the annular ligament during varus stress, but it is not routinely characterised on MRI.

\section{Muscles and tendons}

The muscles around the elbow are considered dynamic stabilisers. The anterior compartment contains the long and short head of the biceps, which inserts distally onto the bicipital tuberosity of the radius, and the brachialis, originating along the anterior surface of the humerus and inserting into the ulnar tuberosity [24-27]. The tendon of the long head of the biceps inserts proximal to the short head and occupies most of the radial tuberosity [28] (Figure 2). The superficial layers of the biceps tendon merge with those of the medial muscle bulk of the proximal forearm at the elbow to form the bicipital aponeurosis (lacertus fibrosus). Studies have shown that this structure can strengthen the distal biceps tendon and help reduce its retraction when ruptured $[29,30]$.
The posterior compartment contains the triceps muscle that inserts distally onto the olecranon with complex footprint anatomy [31]. It is important to note that the medial head of the triceps has a muscular insertion at the olecranon deep to the lateral and long heads $[31,32]$. The anconeus has its origin in the lateral humeral epicondyle and inserts in the olecranon. The anconeus epitrochlearis is an accessory muscle present in a small proportion of individuals, originating from the medial epicondyle and inserting into the olecranon, and it has been associated with ulnar nerve compression [33].

The medial muscle group of the elbow is responsible for flexion-pronation of the forearm and is comprised of six muscles: the pronator teres, flexor carpi radialis (FCR), palmaris longus (PL), flexor digitorum superficialis (FDS), flexor carpi ulnaris (FCU), and flexor digitorum profundus (FDP). Five of these muscles form a common flexor tendon that includes a portion of the pronator teres, the FCR, PL, the humeroulnar head of the FDS, and humeral head of the FCU. The FCU arises from two heads, one originates from the humeral epicondyle and another from the olecranon. A fibrous band, referred to as ligament of Osborne, arcuate ligament, or cubital retinaculum, crosses over these two origins and constitutes the roof of the ulnar cubital tunnel.

The lateral muscle group of the elbow is responsible for the extension-supination of the forearm and consists of another seven muscles: the brachioradialis, extensor carpi radialis longus (ECRL), extensor carpi radialis brevis (ECRB), extensor digitorum (ED), extensor digiti minimi (EDM), extensor carpi ulnaris (ECU), and the supinator. Four of these muscles (ECRB, ED, EDM, and ECU) have a common origin at the lateral epicondyle and form a common extensor tendon. The other components present a complex origin that includes the humeral supracondylar ridge, lateral collateral ligament, and supinator crest of the ulna $[15,19]$.

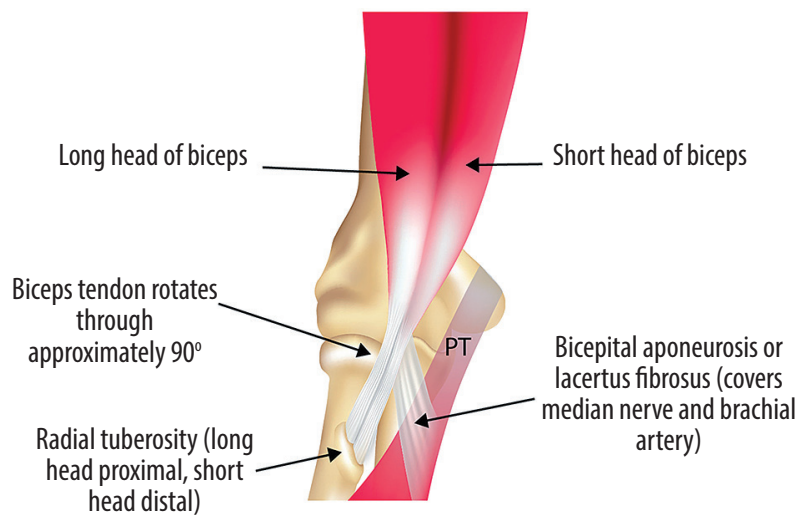

Figure 2. Schematic of the distal biceps tendon showing the long and short head insertions onto the radial tuberosity. The bicipital aponeurosis originates from the distal tendon and expands medially, blending with the fascia of the forearm. PT - pronator teres muscle 


\section{Nerves}

The nerve anatomy in the upper extremity is complex, and a thorough description is beyond the scope of this article. At the level of the elbow there are three primary nerves: the radial, ulnar, and median. The radial nerve passes between the brachial and brachioradialis muscles, bifurcating into superficial sensory and deep motor branches. The deep branch pierces the supinator muscle between its superficial and deep heads, at which point it is also referred to as the posterior interosseous nerve, while the superficial branch continues in the forearm between the brachioradialis and the superficial head of the supinator. The ulnar nerve passes posterior to the medial epicondyle inside the cubital tunnel, which is an anatomic space roofed by Osborne's ligament, or the cubital retinaculum. Finally, the median nerve passes between the lacertus fibrosus and the brachial muscle, continuing between the two heads of the pronator teres muscle in the medial aspect of the forearm [10,34]. Before crossing the elbow, the median nerve passes over the anteromedial aspect of the humerus in the distal arm, where a bone spur, referred to as the supracondylar process of the humerus, can be present in some individuals. This structure may be connected to the medial epicondyle through a ligament (ligament of Struthers), forming a canal that has been associated with median neuropathy [34].

\section{Bursae and plicae}

The distal biceps tendon does not have a tendon sheath. Two bursae can be seen about the distal biceps tendon: the bicipitoradial and the variably present interosseous bursa. The bicipitoradial bursa is located between the distal biceps tendon and the radial bicipital tuberosity and is responsible for reducing friction between these two structures. It may be a cause of pain when distended by fluid, usually in inflammatory arthropathies or mechanical overload $[35,36]$. The interosseous bursa is present in about $20 \%$ of individuals and is located at the medial aspect of the antecubital fossa, between the brachialis muscle and the radius.

The olecranon bursa is located in the subcutaneous region posterior to the olecranon and can be inflamed in the setting of septic and inflammatory arthropathies, trauma, and repetitive stress [25,37].

Synovial plicae or folds are remnants of the embryological formation of the joints [38]. At the elbow they have been described in the posterior, medial, lateral, and anterior compartments as thin intraarticular projections of the synovial capsule in both symptomatic and asymptomatic patients. They can be considered physiological components and only occasionally cause clinical symptoms. The radiocapitellar synovial plica can be particularly prominent and may be associated with joint pain [39].

\section{Magnetic resonance imaging technique and protocol}

The patient can be scanned in the supine position with the arm fully extended, or in the prone position with the shoulder abducted and the arm above the head (the superman position). In the prone position, the elbow stays in the centre of the magnetic field, which can reduce inhomogeneities and artifacts, but this has the disadvantage of being uncomfortable for the patient, eventually leading to motion artifacts. It is essential to always cover the biceps tendon insertion onto the radial tuberosity within the field of view. A conventional MRI protocol commonly includes a combination of non-fat-suppressed T1-weighted, non-fat-suppressed PD-weighted, fat-suppressed T2-weighted, or short tau inversion recovery sequences, in axial, coronal oblique, and sagittal oblique planes [40] (Table 1). It is crucial to first plan the coronal oblique plane using both humeral epicondyles in the axial plane, and then, perpendicular to it, the sagittal and axial planes can be programmed (Figure 3). As stated previously, the distal humerus has an internal rotation compared to the long axis of the humerus, so the coronal oblique plane should be used as a reference for proper visualisation of the tendons and ligaments of the elbow. MRI of the flexed elbow, abducted shoulder, forearm supinated the so-called FABS position - can be used to better show the biceps tendon insertion into the radial tuberosity [41].

Administration of gadolinium-based contrast is not routinely recommended; however, MR arthrography can be helpful to assess intra-articular bodies and stability of osteochondral lesions. Between 5 and $10 \mathrm{ml}$ of total injectate

Table 1. Sample non-contrast elbow imaging protocol at $3 \mathrm{~T}$

\begin{tabular}{|l|c|c|c|c|c|c|}
\hline Sequence & TR $(\mathrm{ms})$ & TE $(\mathrm{ms})$ & TI $(\mathrm{ms})$ & Slice thickness $(\mathrm{mm})$ & FOV $(\mathrm{mm})$ & Matrix \\
\hline Axial T2 FS & 4000 & 65 & & 3.0 & 130 & $320 \times 288$ \\
\hline Coronal T1 & 750 & 10 & & 3.0 & 150 & $384 \times 288$ \\
\hline Coronal STIR & 5900 & 30 & 220 & 3.0 & 150 & $384 \times 288$ \\
\hline Sagittal T1 & 750 & 10 & & 3.0 & 150 & $384 \times 288$ \\
\hline Sagittal T2 FS & 4000 & 65 & & 3.0 & 150 & $384 \times 288$ \\
\hline
\end{tabular}

FOV - field of view, FS - fat-suppressed, PD - proton density, STIR - short tau inversion recovery, TE - echo time, TI - inversion time, TR - repetition time 

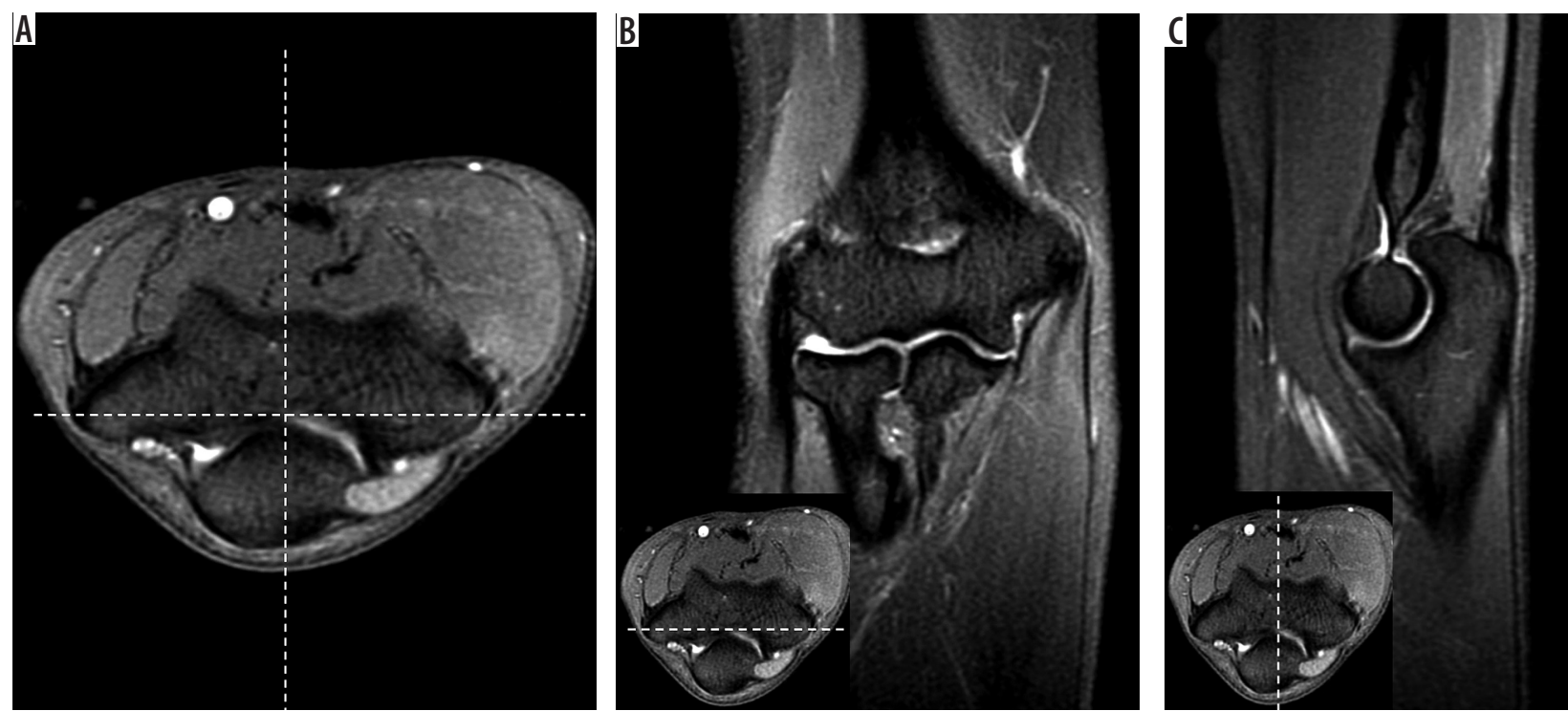

Figure 3. A) Axial, (B) coronal oblique, and (C) sagittal oblique proton density-weighted fat-suppressed magnetic resonance imaging shows typical planning of the imaging planes during the exam. Correlation between axial, coronal oblique, and sagittal oblique planes is shown ( $B$ and $\mathbf{C}$, inset)

should be used with direct MR arthrography using a variety of different approaches [40,42]. The optimum gadolinium concentration for maximal signal-to-noise ratios at 1.5-3T ranges from 0.7 to $3.4 \mathrm{mmol} / \mathrm{l}$ (or less than $2 \mathrm{mmol} / \mathrm{l}$ if iodinated contrast is also used) $[43,44]$. Frequently used protocols include fat-suppressed T1-, PD-, and T2-weighted sequences in the coronal oblique, sagittal oblique, and axial planes. The addition of a non-fat-suppressed sequence can be helpful in a variety of scenarios where the visualisation of fat is desirable, such as for improved boundary delineation for certain tendons and ligaments as well as evaluation of intra-muscular fat. At times it is also useful for tissue characterisation and troubleshooting.

\section{Ligament abnormalities}

Despite being a relatively stable joint, the elbow is the second most commonly dislocated joint in adults, and the most commonly dislocated major joint in the paediatric population [45]. There is a calculated incidence of 5.21 dislocations per 100,000 person-years in the United States, according to a study by Stoneback et al. [46]. Traumatic and sports-related injuries are the most common causes and can lead to chronic joint instability [11]. Physical exam manoeuvres show high sensitivity for most of the injuries about the elbow [47], but pain in the acute setting of trauma or chronic adaptive changes in athletes can reduce its accuracy.

An injured ligament can present on MRI with high signal intensity on fluid-sensitive sequences, wavy contours, or complete discontinuity. Oedema and joint capsule rupture with effusion and fluid extravasation are also usually observed. Conventional MRI has a high sensitivity for complete ligament tears, but only moderate sensitivity for partial tears [48]. MR arthrography, however, has the highest reported accuracy for the evaluation of ligament injuries at the elbow, and can be particularly helpful for medial collateral ligament lesions [49].

At the medial compartment, the anterior bundle of the UCL is the most commonly injured. Although elbow

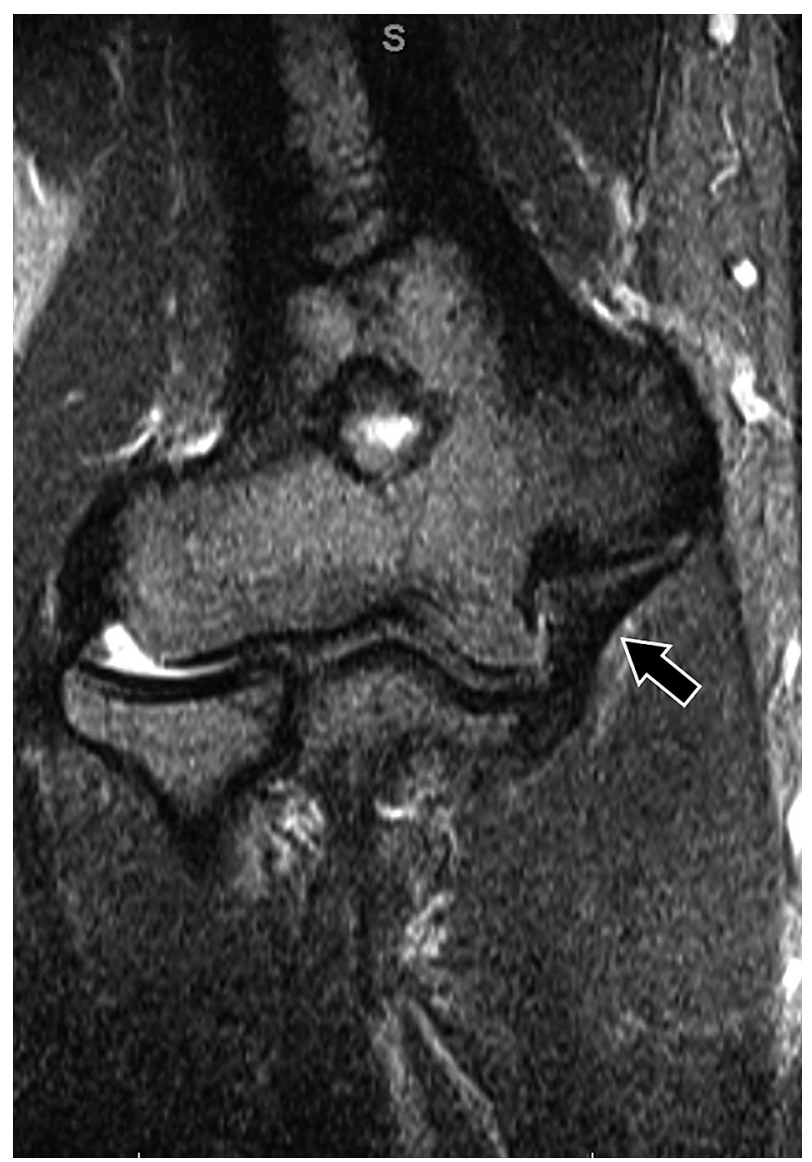

Figure 4. Adaptive changes in throwing elbow. 36-year-old asymptomatic professional baseball pitcher with adaptive ulnar collateral ligament (UCL) thickening. Coronal oblique proton density-weighted fat-suppressed magnetic resonance imaging shows thickening of the anterior bundle of the UCL (arrow) 


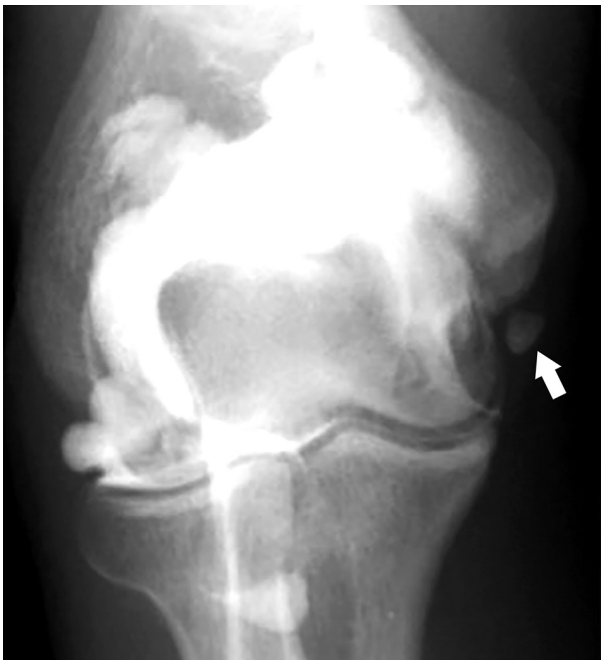

Figure 5. Sequela of chronic ulnar collateral ligament (UCL) injury in a 21-year-old baseball pitcher. AP radiograph from pre-magnetic resonance imaging arthrogram injection shows a well-defined ossicle in the pathway of the anterior bundle of the UCL consistent with chronic trauma (arrow)

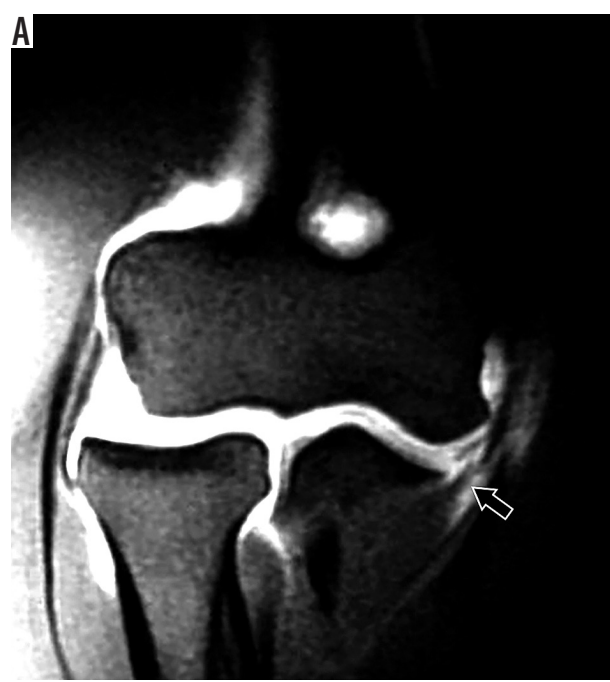

Figure 7. Full-thickness tear of the anterior bundle of the ulnar collateral ligament (UCL) and a prominent synovial fringe in a baseball pitcher. A) Coronal T1-weighted fat-suppressed and (B) short tau inversion recovery magnetic resonance imaging shows extravasation of the intra-articular contrast through the distal aspect of the anterior bundle of the UCL (arrows) consistent with a full-thickness tear. In (B), note a prominent synovial fringe at the lateral aspect of the elbow (arrowhead)

dislocations can cause acute lesions to the UCL, the most common cause of UCL injury is chronic valgus stress from overhead activities, like baseball pitching and javelin throwing. Swimming, gymnastics, and tennis are other sports activities that predispose to UCL injuries [50]. In a recent study of the prevalence of elbow injuries in athletes at the summer Olympic Games, $80 \%$ of lesions included UCL [51]. However, it is difficult to differentiate adaptive asymptomatic abnormalities, typically found in the throwing athlete, from symptomatic findings. One study found that asymptomatic baseball pitchers commonly had ligamentous thickening, bone osteophytes, and tendinopathy in the posteromedial compartment of the elbow [52] (Figure 4). These adaptive changes are caused by repetitive stress across the medial aspect of the joint from overhead throwing. The late cocking and early accel-
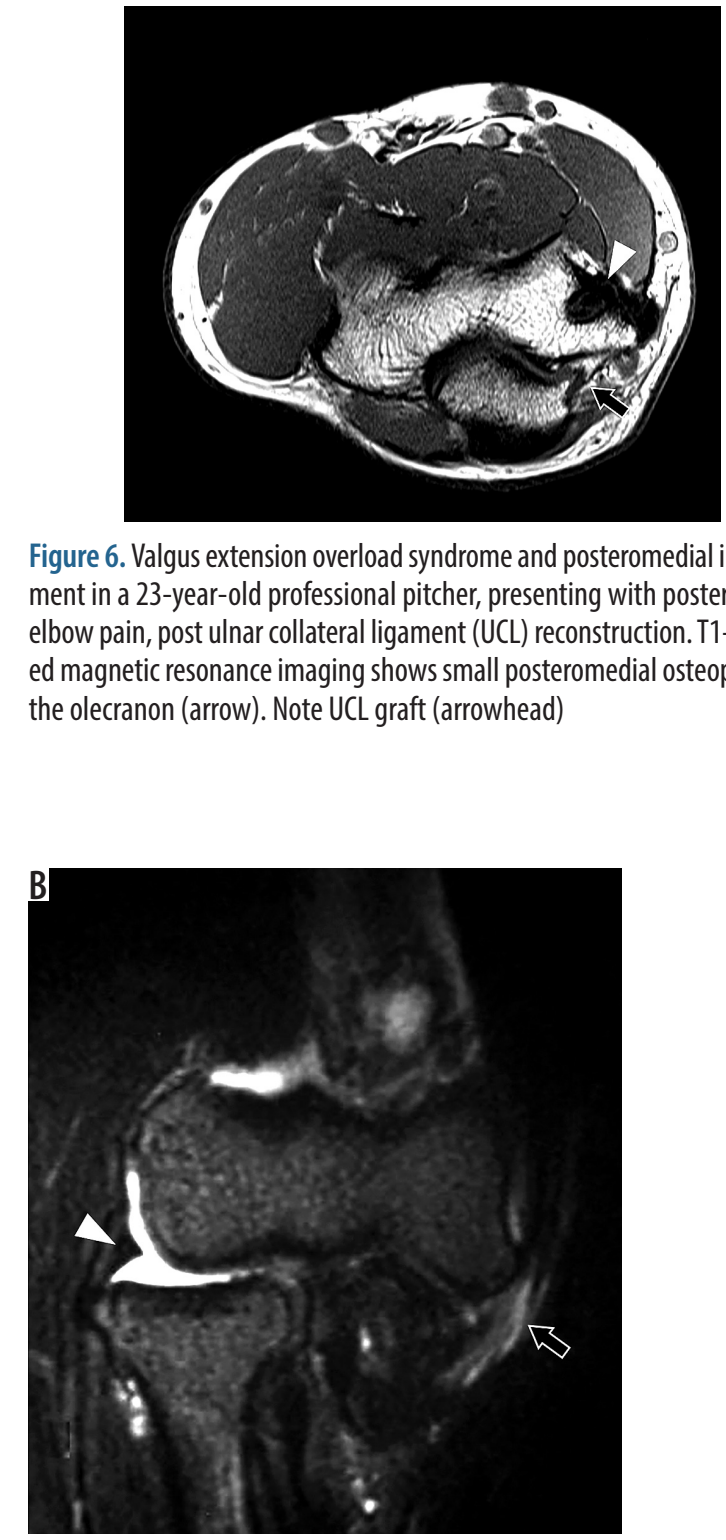

Figure 6. Valgus extension overload syndrome and posteromedial impingement in a 23-year-old professional pitcher, presenting with posteromedial elbow pain, post ulnar collateral ligament (UCL) reconstruction. T1-weighted magnetic resonance imaging shows small posteromedial osteophytes at the olecranon (arrow). Note UCL graft (arrowhead)

eration phases of the throwing movement cause increased tension over the medial compartment of the elbow, high shear stress on the posterior compartment, and compression forces at the lateral compartment, due to transfer of high humeral torque to rapid elbow extension [53].

Repetitive valgus stress leads to chronic degeneration with increased laxity of the UCL and joint instability. Posterior contact between the humerus and ulna leads to the formation of posteromedial bone spurs, cartilage and subchondral bone degeneration, and ultimately osteoarthritic changes. Avulsion fractures of the medial epicondyle and olecranon stress fractures are common findings seen on radiograph and computed tomography (CT). The combination of these abnormalities can lead to decreased elbow terminal extension with posterior elbow pain during ball release, a condition called VEO syndrome or pitcher's 

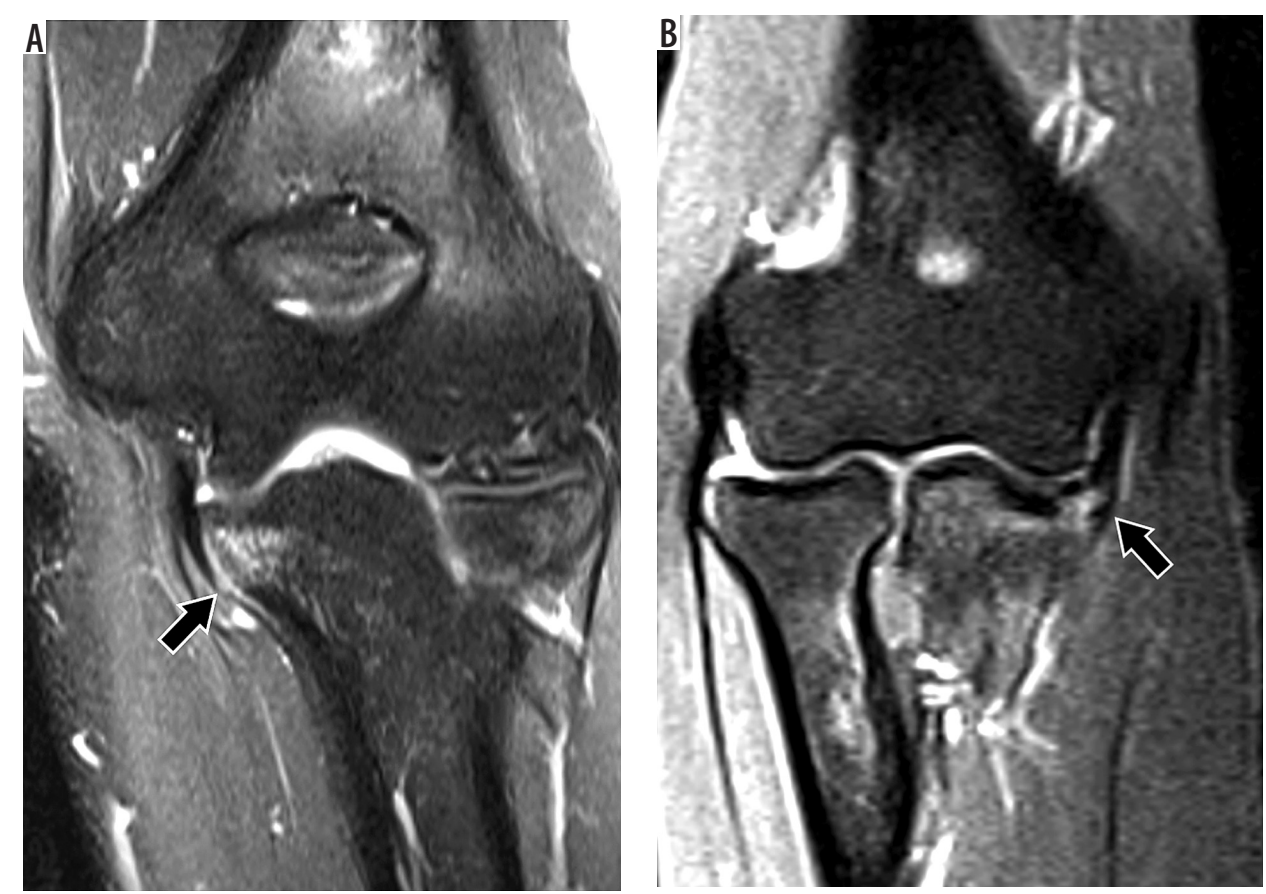

Figure 8. Ulnar collateral ligament (UCL) abnormalities on coronal fluid-sensitive magnetic resonance imaging from different pitchers. A) 16-year-old with stripping and low-grade partial tearing of the anterior bundle of the UCL at the ulna with underlying marrow oedema in the coronoid process (arrow). B) 15-year-old with cortical discontinuity at the sublime tubercle with bone marrow oedema, consistent with an avulsion fracture (arrow)
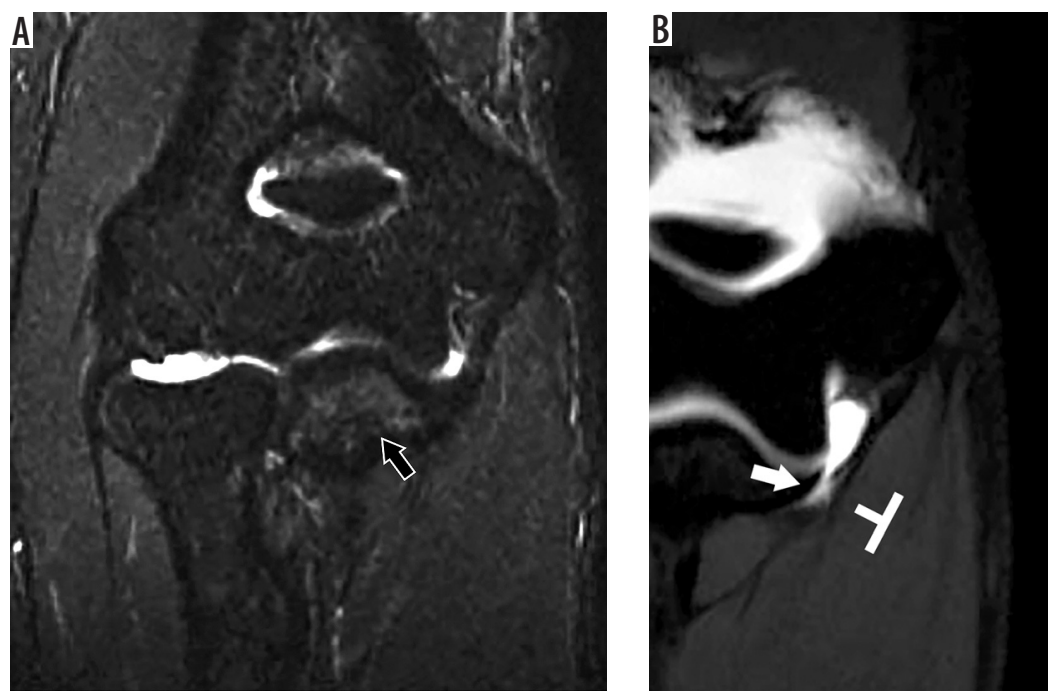

Figure 9. Partial-thickness tear of the anterior bundle of the ulnar collateral ligament (UCL) at the sublime tubercle in an 18-year-old pitcher with medial elbow pain depicted on magnetic resonance imaging (MRI). A) Coronal short tau inversion recovery MRI shows minimal bone marrow oedema in the coronoid process of the ulna consistent with stress reaction (arrow) and no evidence of the UCL tear. B) Coronal T1-weighted fat-suppressed MR arthrography shows contrast insinuating along the medial margin of the sublime tubercle and the anterior bundle of the UCL (arrow) creating T sign

elbow. The differential diagnosis with an acute UCL injury can be inferred by the history and physical exam: the patient with VEO syndrome complains of posterior elbow pain during terminal extension due to impingement, whereas the patient with ULC acute injury refers pain during the beginning of throwing. Physical exam shows the reproduction of pain with valgus stress placed by the examiner onto the elbow while forcing terminal extension. The osseous and soft tissue hypertrophic changes can also lead to ulnar nerve impingement and secondary cubital tunnel syndrome. The examiner must palpate the
Table 2. $0^{\prime}$ Driscoll classification system of posterolateral rotatory instability of the elbow

\begin{tabular}{|l|l|}
\hline Stage & Description \\
\hline 1 & LUCL lesion \\
\hline 2 & LUCL + Anterior joint capsule lesion \\
\hline $3 a$ & LUCL + Anterior joint capsule lesion + MCL posterior bundle lesion \\
\hline $3 b$ & Stage $3 a+M C L$ anterior bundle lesion \\
\hline $3 c$ & Stage $3 b+$ Flexor-pronator muscles disruption \\
\hline
\end{tabular}

LUCL - lateral ulnar collateral ligament, MCL - medial collateral ligament 

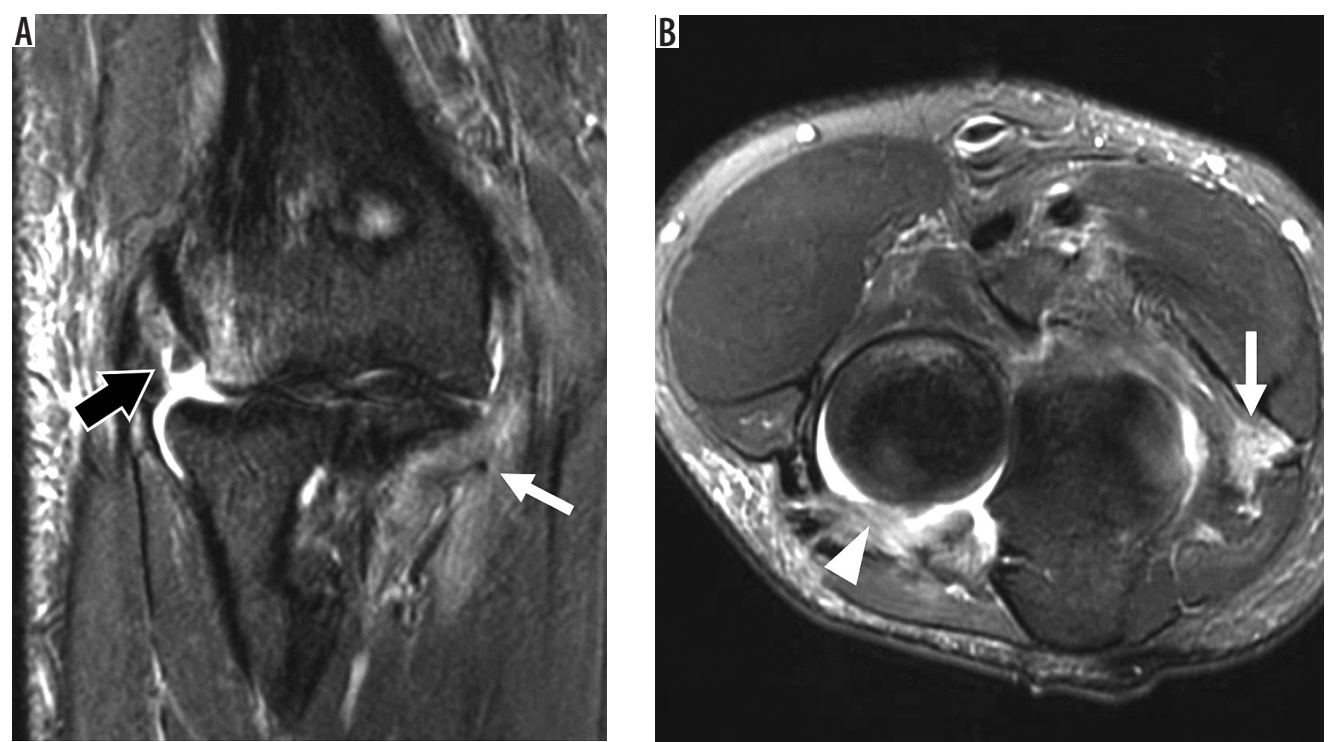

Figure 10. 31-year-old female with 0'Driscoll stage 3 c posterolateral rotatory instability after reduced posterior elbow dislocation. A) Coronal oblique and (B) axial proton density-weighted fat-suppressed magnetic resonance imaging of the elbow shows complete tears of the radial collateral and lateral ulnar collateral ligament (black arrow in A) as well as the annular ligament (arrowhead in B). Oedema is present in the flexor-pronator muscles (thin arrows), indicative of muscle strain

ulnar nerve area during flexion-extension of the elbow, searching for pain or nerve dislocation.

Imaging may show ossification of the UCL (Figure 5), posteromedial humeroulnar osteophytes (Figure 6), osteochondral lesions, avulsions of the medial epicondyle, and sublime tubercle as well as intra-articular bodies. MRI is particularly useful to depict UCL injuries (Figure 7 and 8), which occur most commonly at its proximal and distal insertions, as well as associated tendon and muscle injuries, and joint effusions [54]. MR arthrography has higher sensitivity for partial-thickness ligament tears and may show intra-articular contrast insinuating along the medial margin of the sublime tubercle and under the UCL (called T sign due to similarity with the letter T lying on its side) (Figure 9). It is important to remember that the ulnar footprint of the UCL can be several millimetres distal to the articular margin, which can be an imaging pitfall for ligament injuries $[16,55,56]$. Treatment of VEO syndrome initiates with conservative measures like active rest, physiotherapy, and non-steroid anti-inflammatory medication but may require arthroscopy with osteophyte removal and medial corner olecranon osteotomy in refractory cases [50].

Treatment of partial UCL tears is initially conservative with active rest, non-steroidal anti-inflammatory drugs (NSAIDs), rehabilitation, and strengthening exercises. Overhead throwing athletes with complete disruption of the anterior bundle of the UCL or those with incomplete tears but who are unable to return to competitive throwing may require surgical ligament reconstruction, which can be done through several techniques, the most famous been the Tommy John surgery, named after the baseball pitcher Tommy John, and first performed by doctor Frank Jobe in 1974.
The LCL is less frequently injured compared to the UCL, with traumatic joint subluxation/dislocation as the most common aetiology. Attention should be paid to the LUCL because it is particularly important for maintaining lateral elbow stability. LUCL injury can present with attenuation, signal alteration, thinning, and complete discontinuity.

Posterolateral rotatory instability of the elbow (PLRI) is a condition first described by O'Driscoll et al. [3], caused by a lesion of the LUCL that leads to posterolateral subluxation of the radius on the capitellum. The injury mechanism is a fall onto an outstretched hand with the shoulder abducted, associated with forearm axial loading, external elbow rotation, and valgus overload. Later, O'Driscoll et al. described a classification system for better clinical staging and treatment [57] (Table 2). This classification is based on the grade of elbow instability according to the increasing injury pattern. First, there is a lesion of the LUCL, with or without RCL injury, which leads to posterior subluxation of the elbow. An additional anterior joint capsule injury causes increased posterolateral subluxation. The final stages of PLRI include lesions of the UCL and flexor-pronator muscle bulk, with consequently gross joint instability [11] (Figure 10). A specific finding associated with PLRI is a compression fracture in the posteroinferior aspect of the capitellum, caused by subluxation-dislocation of the posterior radial head [9]. The impaction between the two articular surfaces is similar in pattern to the anterior glenoid bone defect seen in anterior shoulder dislocation, and it has been referred to as the Osborne-Cotterill lesion [58] (Figure 11). Patients complain of chronic pain and a clicking sensation. Surgical treatment options include LUCL repair or ligament reconstruction, depending on chronicity and tissue status. 

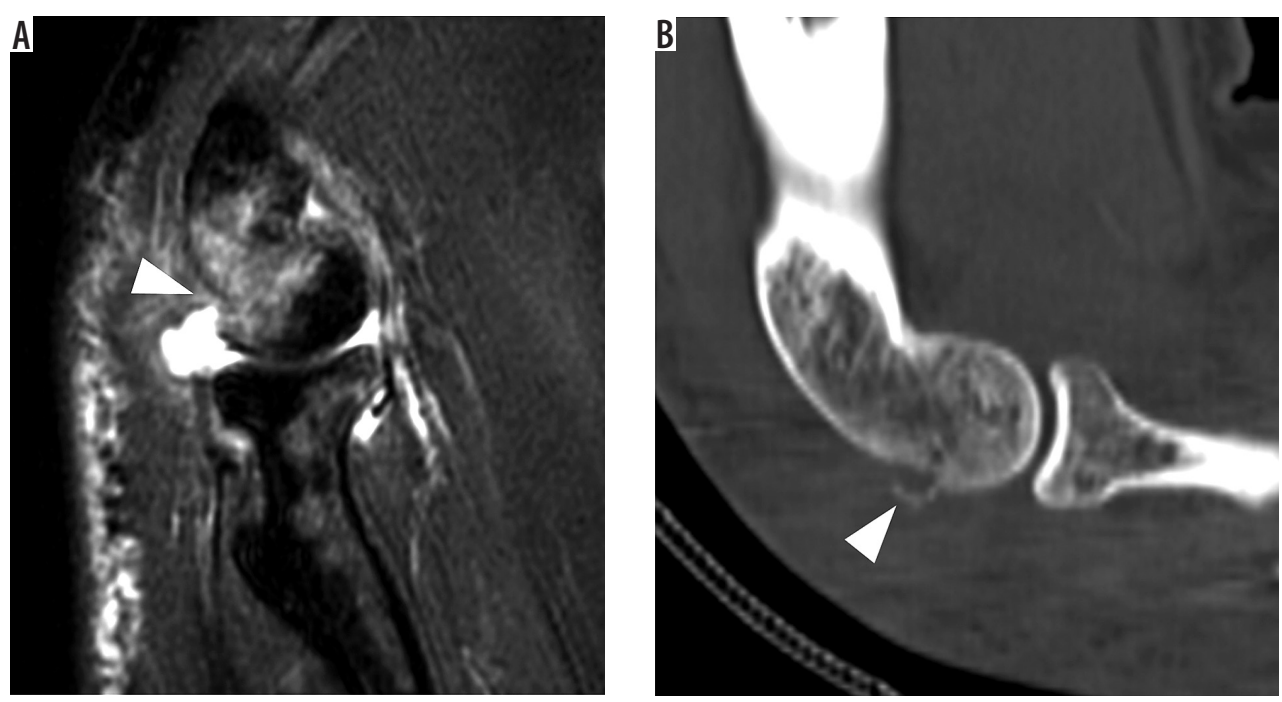

Figure 11. Osborne-Cotterill lesion characteristic of posterolateral rotatory instability. A) Sagittal oblique proton density-weighted fat-suppressed magnetic resonance imaging post-reduction of a posterior elbow dislocation shows an impaction fracture of the posterior aspect of the capitellum with associated bone marrow oedema (arrowhead), known as an 0sborne-Cotterill lesion. B) Sagittal reformatted computed tomography image confirms the impaction and better depicts small fracture fragments (arrowhead)

In children, a common lesion is the subluxation or dislocation of the radial head from the annular ligament with the traction of the forearm and hand, which is also referred to as the nursemaid's elbow. Conservative treatment is generally indicated unless there is ligament displacement and interposition within the humeroradial joint, which may require operative treatment [23]. In adults, the annular ligament is commonly involved in elbow trauma and dislocation and is usually associated with other ligament lesions. Ligament detachment and interposition in the radiocapitellar joint have also been described, although isolated tears are uncommon.

\section{Tendon abnormalities}

Tendinopathy is caused by chronic repetitive stress or degeneration that leads to microavulsions at the tendon insertion. In the initial tendinosis MRI shows thickening and intermediately increased signal intensity on T1- and T2-weighted sequences, corresponding histologically to collagen degeneration and neovascularisation. As the disease progresses MRI shows fluid-like signal intensity changes within the tendon on T2-weighted images, indicating collagen disruption, which may evolve to tendon thinning (partial tear) or a complete tear. It is crucial to consider the magic angle effect, which is an MRI artifact related to anisotropy, especially evident in high collagencontaining tissues. The magic angle effect is maximally present when the long axis of the structure is oriented at approximately $54^{\circ}$ to the main magnetic field, and increased signal intensity may be evident, particularly on short TE sequences (such as T1-weighted images). In these situations, the longer TE sequences (such as fatsuppressed T2-weighted images) should be used to more accurately characterise abnormalities [59]. In addition,
MRI can show secondary findings associated with tendon pathology, such as bone marrow oedema and periostitis.

Tendon overuse, characterised by mechanical stress with an inadequate healing/adaptation response, can result in a tendinopathy. When it involves the common extensor tendon, it is referred to as lateral epicondylosis, or tennis elbow (Figure 12), and when it involves the common flexor tendon it is referred to as medial epicondylosis, or golfer's elbow (Figure 13). Lateral epicondylosis is the most common tendinopathy about the elbow, and the most common cause of elbow pain in non-athletes, presenting as a chronic burning pain at its lateral aspect. It may be associated with LCL injury, particular the LUCL origin at the lateral epicondyle. Studies have

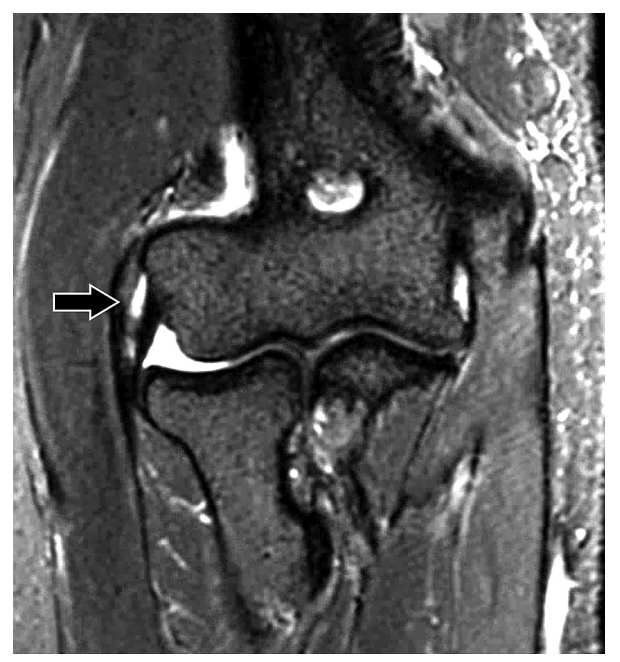

Figure 12. 38-year-old man with lateral epicondylosis. Coronal oblique proton density-weighted fat-suppressed magnetic resonance imaging shows a moderate-grade partial-thickness interstitial tear of the common extensor tendon origin (arrow). Note associated mild thickening of the subjacent proximal radial collateral ligament 

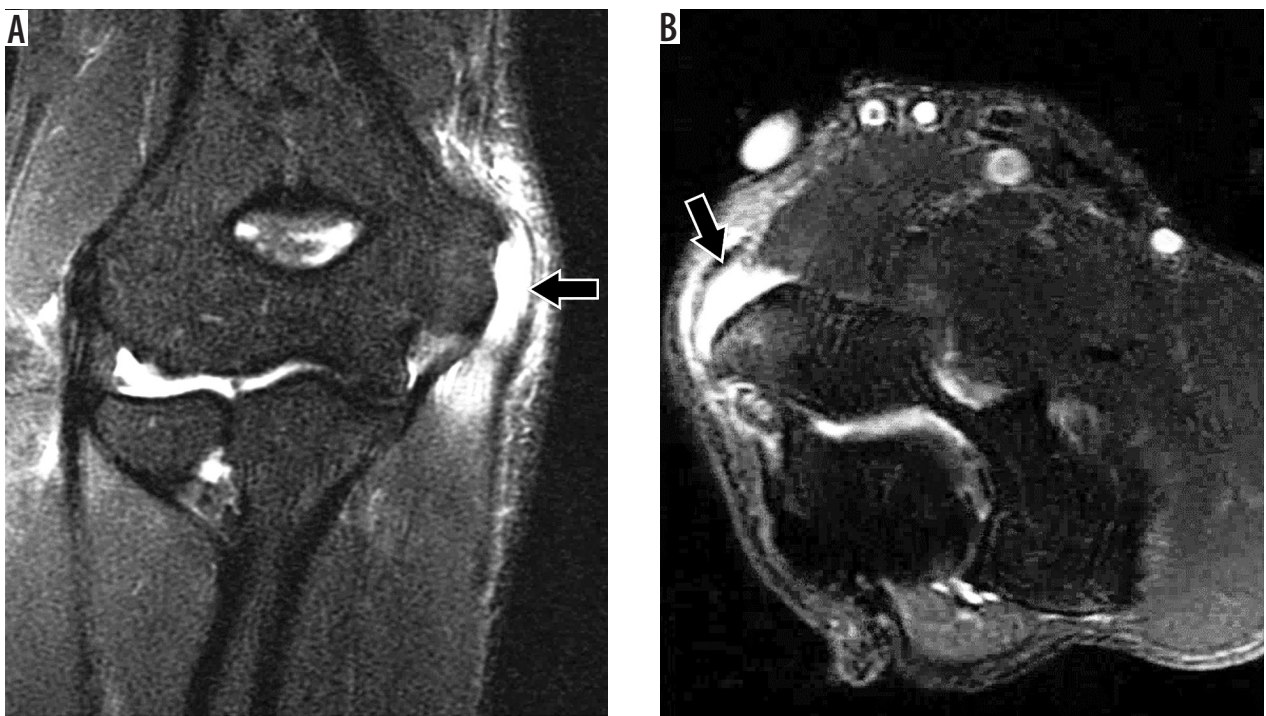

Figure 13. 41-year-old woman with medial epicondylosis. A) Coronal oblique and (B) axial proton density-weighted fat-suppressed magnetic resonance imaging shows a high-grade partial-thickness tear of the common flexor tendon origin (arrows). There is associated subjacent bone marrow oedema and surrounding soft tissue oedema
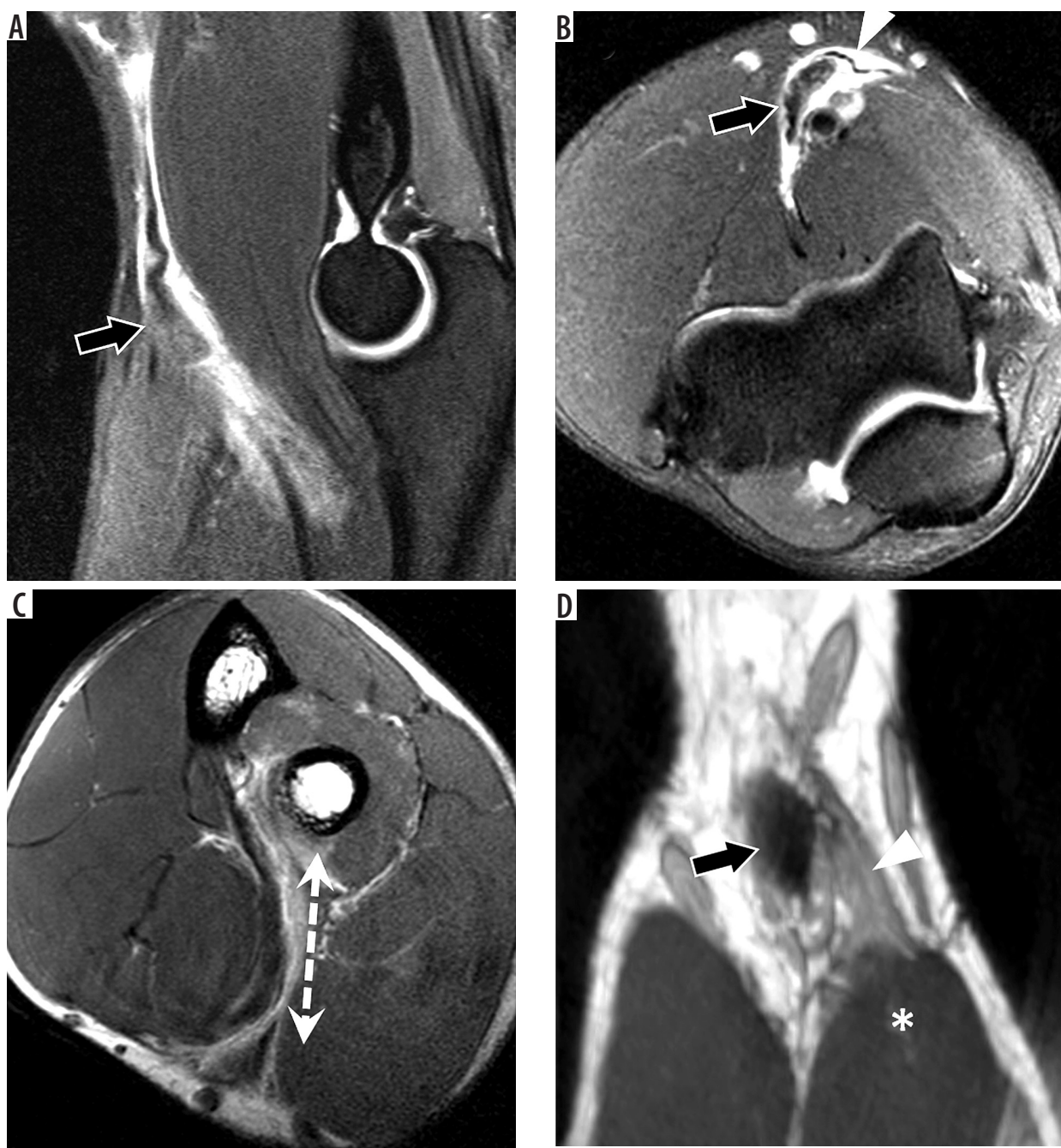

Figure 14. 31-year-old man with distal biceps tendon rupture. A) Sagittal oblique and (B) axial proton density (PD)-weighted fat-suppressed magnetic resonance imaging (MRI) shows rupture and retraction of the distal biceps tendon (arrows) with surrounding soft tissue oedema. The lacertus fibrosus is partially torn (arrowhead in B). C) Flexed elbow, abducted shoulder, forearm supinated positioning MRI confirms distal biceps tendon rupture with $4 \mathrm{~cm}$ of retraction (dashed line). D) Coronal MRI reformatted from 3D PD-weighted fast-spin echo dataset shows the partially intact lacertus fibrosus (arrowhead) connecting the biceps tendon (arrow) to the flexor musculature (asterisk) 
suggested an association between lateral epicondylosis and mild lateral elbow instability [60]. Medial epicondylosis is much less frequent than lateral epicondylosis, being most commonly associated with chronic stress from sports activities like overhead throwing. Treatment for tendinopathies are generally conservative, consisting of physical therapy, analgesics, and rest, but in refractory disease, percutaneous interventions or surgical treatment may be indicated $[25,61]$.

Complete rupture of the distal biceps tendon can be observed on MRI as a discontinuity and proximal retraction, sometimes associated with bicipitoradial bursitis. It is important to remember that the long and short heads of the biceps tendon can have distinct insertions into the radial tuberosity, and thus both components should be evaluated on MRI $[24,62,63]$. Intact bicipital aponeurosis, or lacertus fibrosus, may prevent proximal tendon retraction when the biceps is ruptured, which may cause underestimation of biceps tendon tear clinically and on MRI (Figure 14).

Triceps tendon ruptures are rare but usually occur at the insertion site. MRI may show discontinuity of the long and lateral heads (Figure 15). The deep medial head inserts directly onto the olecranon and is rarely torn [31].

\section{Osseous and chondral lesions}

MRI is crucial for the diagnosis and characterisation of bone and cartilage lesions at the elbow, showing high sensitivity for subchondral bone damage, intraarticular bodies, and stress fractures. Children and athletes are two groups commonly affected due to skeletal immaturity and chronic repetitive stress, respectively. It is, therefore, essential to understand the particular developmental anatomy of the elbow [64].

The ossification centres and apophyses in the immature skeleton have lower stability than the mature adult skeleton due to the weaker mechanical properties of unossified cartilage. Also, the physes can be under tremendous tensile forces, which make them more vulnerable to trauma. The elbow has six ossification centres: capitellum, radial head, medial epicondyle, trochlea, olecranon, and lateral epicondyle, which appear in that order, by ages 1-56-8-10-11 years, respectively (Table 3 ). They appear and ossify earlier in girls than in boys [64].

Osteochondritis dissecans is a condition of the subchondral bone and articular cartilage occurring in different joints, which is characterised by a localised failure of normal ossification process, leading to fragmentation of the articular surface, instability, and eventually fragment detachment. In the elbow, this condition most commonly occurs at the anterolateral aspect of the capitellum, typically in young adolescents 11-15 years old, involved in throwing sports. The cause is unknown but probably involves chronic repetitive trauma and impaired blood supply leading to disruption of normal endochondral ossification [65].

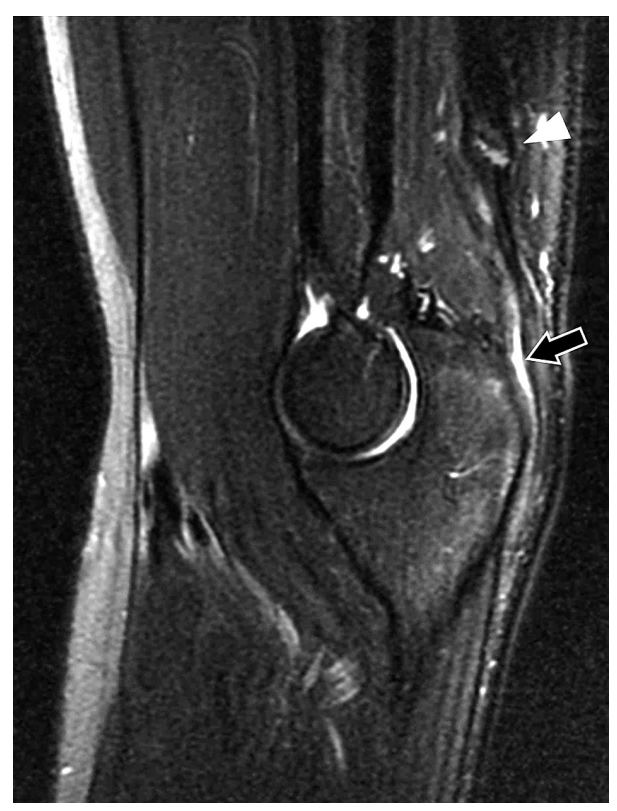

Figure 15. 28-year-old man with triceps tendon rupture. Sagittal oblique proton density-weighted fat-suppressed magnetic resonance imaging shows a high-grade tear of the conjoined tendon of the lateral and long heads of the triceps with proximal retraction (arrow). An associated osseous avulsion fragment is evident in the retracted tendon stump with oedema (arrowhead)

The typical MRI appearance is bone marrow oedema and subchondral cysts. Unstable injuries can be distinguished from stable injuries by fluid interposed between the osteochondral fragment and the underlying bone for in situ lesions (Figure 16), or frank displacement of the fragment. Other signs suggestive of instability are subchondral and medullary cysts around the fragment [66].

Panner first described osteochondrosis of the capitellum or Panner's disease in 1927, in a series of cases of lateral elbow pain in young boys. Laurent and Lindstrom later described similar cases [67]. Although the causes may be multifactorial, Panner's disease is now recognised as a type of osteonecrosis of the capitellum that occurs in boys from 4 to 10 years, therefore earlier than in osteochondritis dissecans, and is usually associated with sports-related elbow valgus overload. Similar to other osteochondroses like Legg-Calvé-Perthes or OsgoodSchlatter, Panner's disease occurs in developing bone and

Table 3. Ossification centres at the elbow and their respective age of appearance

\begin{tabular}{|l|c|}
\hline Ossification centre & Age of appearance (years) \\
\hline Capitellum & 1 \\
\hline Radial head & 5 \\
\hline Medial epicondyle & 6 \\
\hline Trochlea & 8 \\
\hline Olecranon & 10 \\
\hline Lateral epicondyle & 10 \\
\hline
\end{tabular}



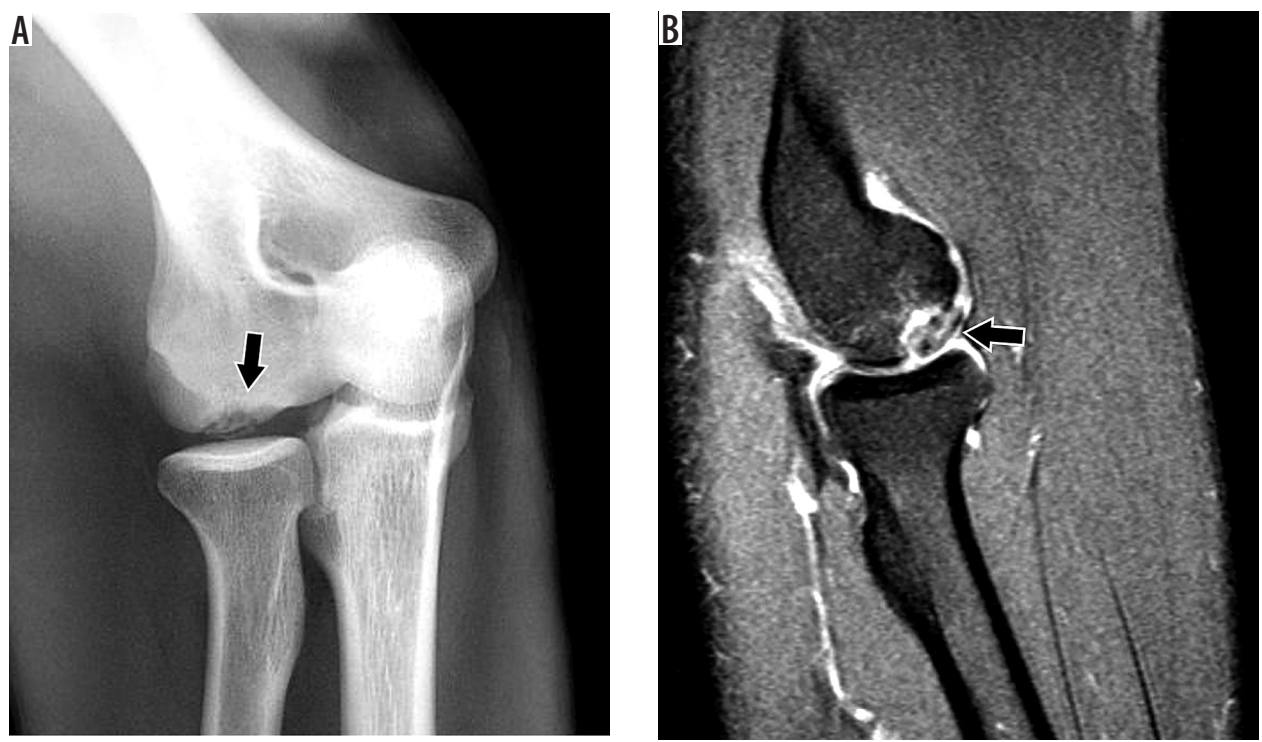

Figure 16. 16-year-old male basketball player with osteochondritis dissecans of the capitellum. A) Anteroposterior radiograph of the elbow shows subchondral lucency in the capitellum (arrow). B) Sagittal oblique proton density-weighted fat-suppressed magnetic resonance imaging confirms the osteochondral lesion characterised by an unstable in situ fragment with bone marrow oedema and subjacent fluid (arrow)

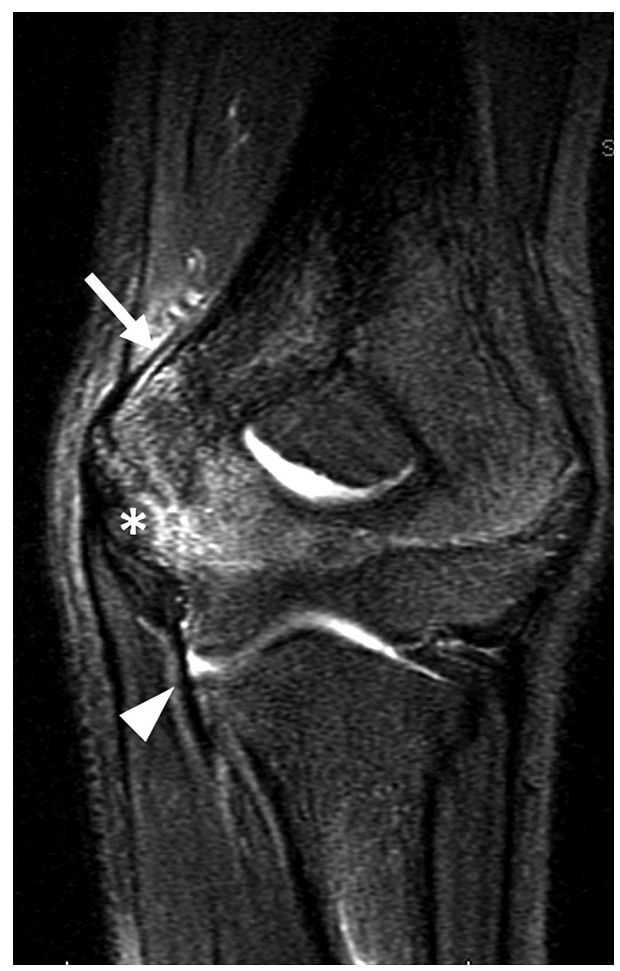

Figure 17. Little leaguer's elbow. Coronal proton density-weighted fat-suppressed magnetic resonance imaging of a 15 -year-old boy shows bone marrow oedema centred in the medial epicondylar apophysis (asterisk), extending to adjacent distal humerus with periostitis (arrow). Note intact ulnar collateral ligament (UCL) (arrowhead) and absence of bone avulsion (no fluid interposed between UCL and sublime tubercle of the ulna or between the apophysis and medial humeral epicondyle)

may present with sclerosis, characterised by low signal intensity on all MRI sequences, along with oedema and joint effusion on fluid-sensitive sequences. Usually there is no bone fragment, and the cartilage remains intact, helping to further differentiate it from osteochondritis dissecans.
Table 4. Mason classification of radial head fractures

\begin{tabular}{|l|l|}
\hline Type & Injury \\
\hline I & Undisplaced \\
\hline II & Displaced partial articular \\
\hline III & Multifragmented/Displaced total articular \\
\hline
\end{tabular}

Table 5. Rayes and Morrey classification of coronoid fractures

\begin{tabular}{|l|l|}
\hline Type & Injury \\
\hline I & Coronoid tip fracture \\
\hline II & Fracture less than $50 \%$ of height of coronoid \\
\hline III & More than $50 \%$ of height of coronoid \\
\hline
\end{tabular}

The condition is self-limited, and conservative treatment is recommended.

Medial epicondyle apophysitis, also known as little leaguer's elbow, due to its high incidence in adolescent baseball pitchers, is a disease caused by repetitive stress over the immature bone and physis at the medial elbow. As in the adult athlete involved in an overhead throwing activity, this condition is related to the late cocking and early acceleration phases of overhead throwing, which leads to chronic bone microtrauma and capsuloligamentous injuries. In children, the characteristics of the immature bone explain the higher frequency of medullary and physeal changes compared to ligamentous injuries. Bone marrow oedema and increased physeal width on fluidsensitive sequences are seen on MRI [68] (Figure 17).

Elbow fractures are frequently associated with joint subluxation or dislocation. There are specific classifications for radial head, coronoid, olecranon, and distal humeral fractures. Still they regularly occur simultaneously depending on the mechanism of injury [69], and 

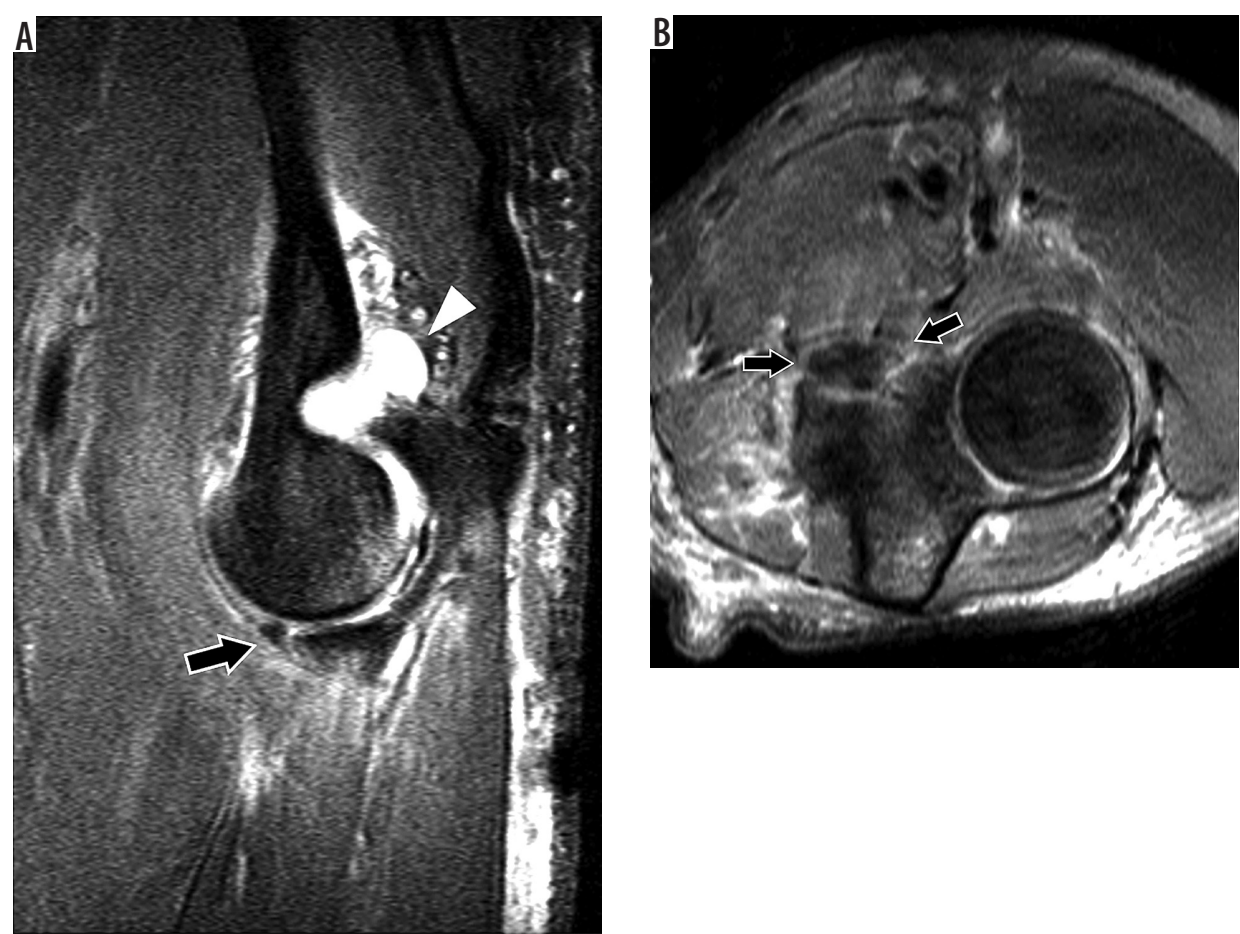

Figure 18. 30-year-old man with elbow dislocation after a fall. A) Sagittal oblique and (B) axial proton density-weighted fat-suppressed magnetic resonance imaging shows a non-displaced fracture of the anteromedial facet of the coronoid process (arrows), consistent with posteromedial rotatory instability. Also present is a joint effusion (arrowhead in A) with periarticular soft tissue oedema

very often the orthopaedic surgeon has to choose the best treatment option based on the combination between them and the instability pattern they cause. CT is the most important exam for evaluation of fracture location and extension, but MRI can be helpful, particularly with nondisplaced fractures or stress fractures [70]. MRI is also particularly useful for evaluation of stress reactions. On MRI, fracture lines are visualised as low signal intensity lines on all pulse sequences with abundant bone marrow oedema on fluid-sensitive sequences.

Radial head fractures are frequently classified using the Mason system (Table 4), which considers the grade of comminution and displacement of bone fragments, with higher grades showing increased comminution and instability. Type I (undisplaced) fractures can be treated conservatively, while type II (partially displaced) and III (comminuted) fractures require operative management [71,72].

Coronoid fractures are frequently classified using the Regan \& Morrey system (Table 5), which takes into consideration the height of the coronoid bone fragment. Larger fragments (involving $50 \%$ or more of the coronoid process height) are associated with poorer prognoses and should be surgically fixated.

The combination of a radial head fracture, ulnar coronoid fracture, and posterior elbow dislocation is referred to as the terrible triad of the elbow, because it is associated with increased elbow instability, complications, and poor prognosis even after operative treatment. A high-force trauma usually causes this type of complex fracture, and the injury mechanism is axial load to an extended and
Table 6. O'Driscoll classification of anteromedial olecranon fractures

\begin{tabular}{|l|l|}
\hline Type & Description \\
\hline I & Anterior rim \\
\hline II & Anteromedial rim (including sublime tubercle) \\
\hline III & Base of olecranon \\
\hline
\end{tabular}

supinated elbow. The proximal ulna and radial head dislocate posteriorly in relation to the humerus. There are also lateral and medial collateral ligament and tendon injuries. Terrible triad injuries do not have any specific classification system, but treatment follows individual classification systems for radial head and coronoid process fractures. Operative treatment with surgical fracture fixation with or without ligament reconstruction is usually necessary.

A distinct type of coronoid process fracture involving the anteromedial rim (or facet) is associated with VPMRI of the elbow and is accompanied by lesions of the LUCL and the posterior bundle of the UCL (Figure 18). The mechanism of injury is a fall onto an outstretched hand, with subsequent internal rotation of the elbow and varus overload. The anteromedial facet of the coronoid process is a medial extension of the ulnar articular surface and plays an essential role in the varus stability of the elbow. A fracture at this site leads to humeroulnar joint incongruity and subsequently osteoarthritis. The larger the osseous fragment, the more likely the development of osteoarthritis if not surgically fixated [73,74]. For this specific type of coronoid fracture, O'Driscoll described a classification system that considers the fracture exten- 

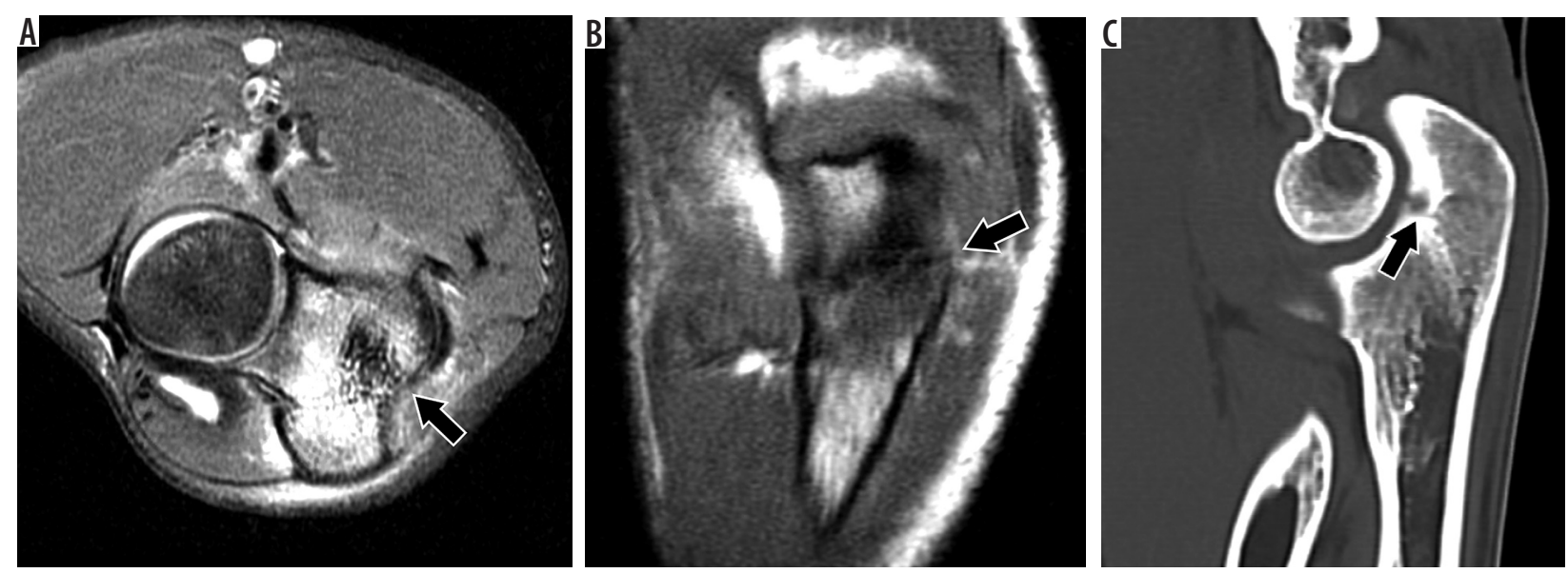

Figure 19. 18-year-old male pitcher with an olecranon stress fracture. A) Axial proton density-weighted fat-suppressed and (B) coronal T1-weighted magnetic resonance imaging shows an olecranon stress fracture (arrows) with associated bone marrow and soft tissue oedema. C) Sagittal reformatted computed tomography image confirms the non-displaced olecranon stress fracture (arrow)

sion from those involving only the anterior rim of the coronoid process to those also involving the anteromedial facet and the sublime tubercle (Table 6). In general, these fractures are treated operatively, although some cases of type I fractures with small bone fragments may be treated with lateral ligament repair only, whereas types II and III always require bone fragment fixation [69].

Olecranon fractures are relatively common and associated with high-energy trauma in adults and low-energy trauma in the elderly [75]. There is some debate over the best classification system: of the several proposed classification systems, the Mayo classification is the most commonly used; however, criticism and controversy exist in the literature [76]. The grade of comminution is the most important feature.

Olecranon stress fractures may occur in the throwing athlete, either due to the pull of the triceps or from impingement in the olecranon fossa (Figure 19) in the setting of VEO syndrome. In adolescent baseball pitchers, medial supracondylar stress fractures can also rarely occur [77]. Of note, stress fractures about the elbow occur less frequently than ligament injuries [78].

Distal humeral fractures occur in young adults submitted to high-energy trauma or in the osteoporotic elderly population. The recommended treatment is operative, and it is based most commonly on the AO classification, which divides fracturs types into groups A (extra-articular), B (partial intra-articular), and C (complete articular) with subdivisions according to the grade of comminution [79].

\section{Neuropathies}

Nerves can be seen on MRI as small tubular structures with low to intermediate signal intensity on T1-weighted images and intermediate to high signal intensity on fluidsensitive sequences. The elbow has three primary nerves (radial, ulnar, and median), and a thorough understanding of anatomy is essential for the evaluation of common neuropathies.

\section{Radial nerve}

Radial tunnel syndrome is a condition characterised by pain at the lateral aspect of the elbow and forearm, in the area involving the proximal extensor muscles, without motor deficit, caused by compression of the radial nerve within the radial tunnel, and worsened by pronation-supination of the forearm. The radial tunnel is an anatomic space between the brachioradialis, brachialis, and ECRB muscles and the capitellum, which extends from the capitellum to the lower portion of the supinator [80]. Potential confounders are lateral epicondylosis and distal biceps tendinopathy [74]. One important clinical finding that can help is that pain associated with radial tunnel syndrome usually occurs distal to the lateral epicondyle. MRI findings may show denervation changes involving both the proximal extensor muscle group (like the triceps, anconeus, and brachioradialis) and the distal extensor muscle group, characterised by muscle oedema in acute and subacute stages, and muscle atrophy in advanced stages.

After passing the lateral epicondyle, the radial nerve bifurcates into a superficial sensory branch that gives sensation to the anterolateral forearm, and a deep motor branch that continues distally to the dorsum of the wrist and innervates the common extensor muscles at the forearm [81]. Once it pierces the supinator muscle the deep motor branch is called the posterior interosseous nerve (PIN), which can be compressed at several sites along its trajectory giving rise to PIN syndrome. The most common site of compression is at the proximal edge of the supinator, between its two heads, by a tendinous structure known as the arcade of Frohse. Other possible points of compression are the medial edge of the ECRB, the recurrent radial vessels (known as the leash of Henry), and the inferior margin of the supinator muscle [82].

PIN syndrome is a neuropathy characterised by pain and muscle weakness at the lateral aspect of the forearm and hand [83], which differentiates it from radial tunnel 

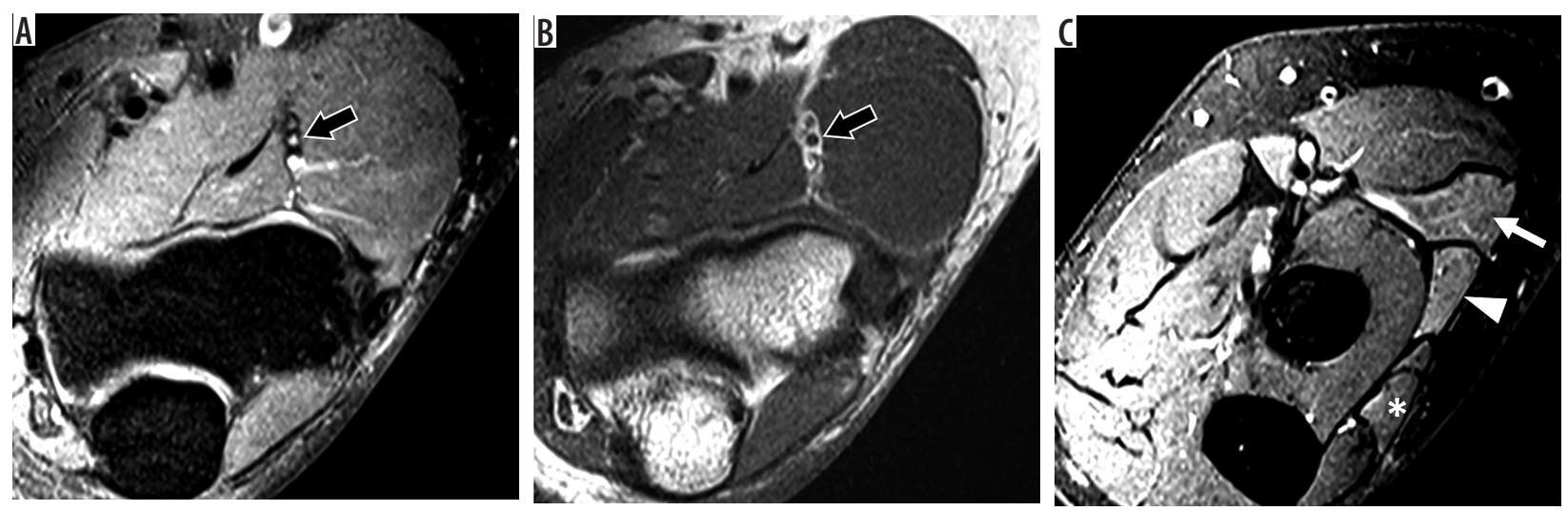

Figure 20. 50-year-old female with history of radial neuropathy. A) Axial proton density (PD)-weighted fat-suppressed and (B) T1-weighted magnetic resonance imaging (MRI) shows the deep branch of the radial nerve (arrows), which is hyperintense on the fluid-sensitive sequence (A). C) Axial PD-weighted fat-suppressed MRI shows muscle denervation changes in the extensor carpi radialis brevis (thin arrow), extensor digitorum (arrowhead), and extensor carpi ulnaris (asterisk), all innervated by the posterior interosseous nerve
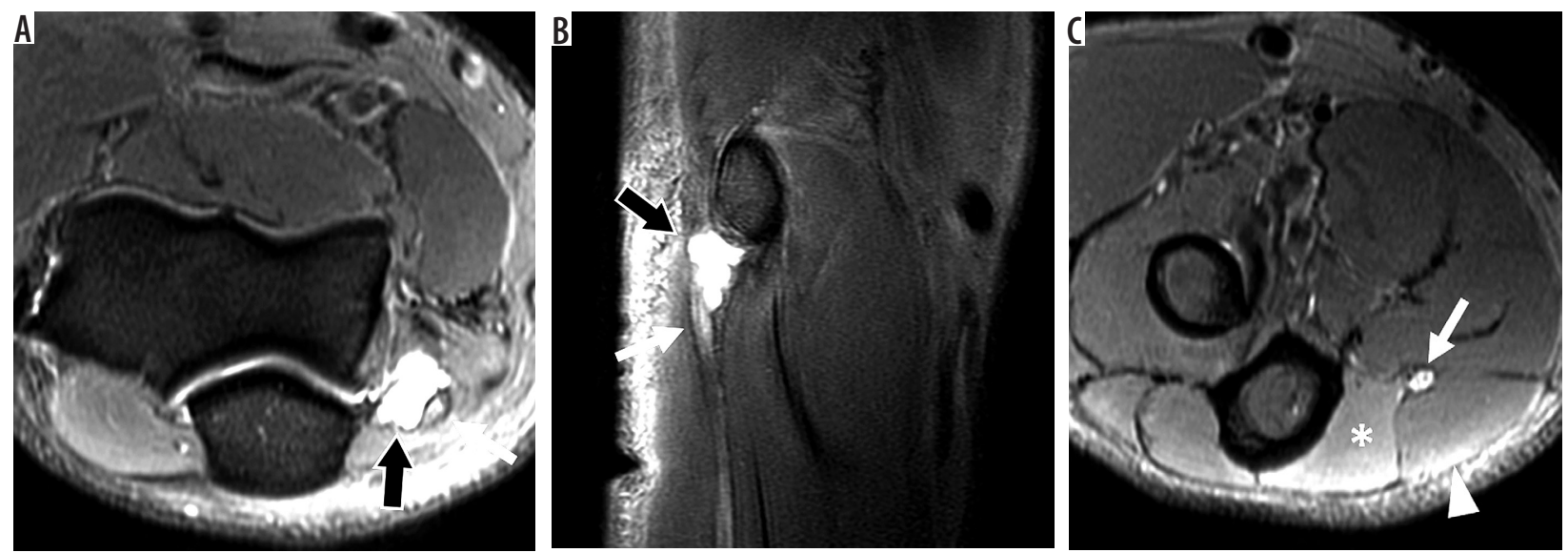

Figure 21. 52-year-old man with cubital tunnel syndrome caused by a ganglion cyst. A) Axial and (B) sagittal proton density-weighted fat-suppressed magnetic resonance imaging (MRI) shows a ganglion cyst (arrows) in the cubital tunnel adjacent to the ulnar nerve which demonstrates mildly increased size and signal. C) Axial proton density-weighted fat-suppressed MRI shows mild oedema in the flexor digitorum profundus (asterisk) and flexor carpi ulnaris (arrowhead) muscles without atrophy adjacent to mildly hyperintense ulnar nerve (arrow)

syndrome that has classically been described as causing pain without motor deficit. There is no sensory deficit, and electrodiagnostic testing may be normal. MRI can show features of distal muscle denervation, in this case involving only the distal common extensor muscle group and the supinator muscle, characterised by high signal intensity on fluid-sensitive sequences, and atrophy with fatty infiltration in T1-weighted images (Figure 20).

The superficial branch of the radial nerve can also be compressed at the distal forearm, most commonly at the posterior border of the brachioradialis tendon, as the nerve transitions from a deep to a subcutaneous location through the fascia that binds the brachioradialis tendon to the ECRL tendon, usually during pronation. Such compression may cause burning pain and paraesthesia at the dorsum of the thumb and radial side of the hand, a condition called Wartenberg's syndrome [84]. Other descriptive names have been used like handcuff neuropathy, wristwatch neuritis, and cheiralgia parestetica due to its simi- larity with compression of the lateral femoral cutaneous nerve at the tight (meralgia paraestetica).

Compression of the radial nerve and its branches can be caused by ganglion cysts, bicipitoradial bursitis, trauma with radial head dislocation, repetitive movements of supination and pronation, infectious diseases, and tumours [85-87]. Treatment of non-tumoral causes begins with immobilisation, analgesics, and physiotherapy, followed by surgical decompression in refractory cases, which has shown good clinical outcomes [88].

\section{Ulnar nerve}

Ulnar neuropathy is the second most common peripheral nerve compression syndrome. It usually occurs at the level of the cubital tunnel, an anatomical space bounded by the medial epicondyle medially and olecranon laterally, the Osborne ligament as its roof, and the posterior bundle of the medial collateral ligament and joint capsule as its floor. 

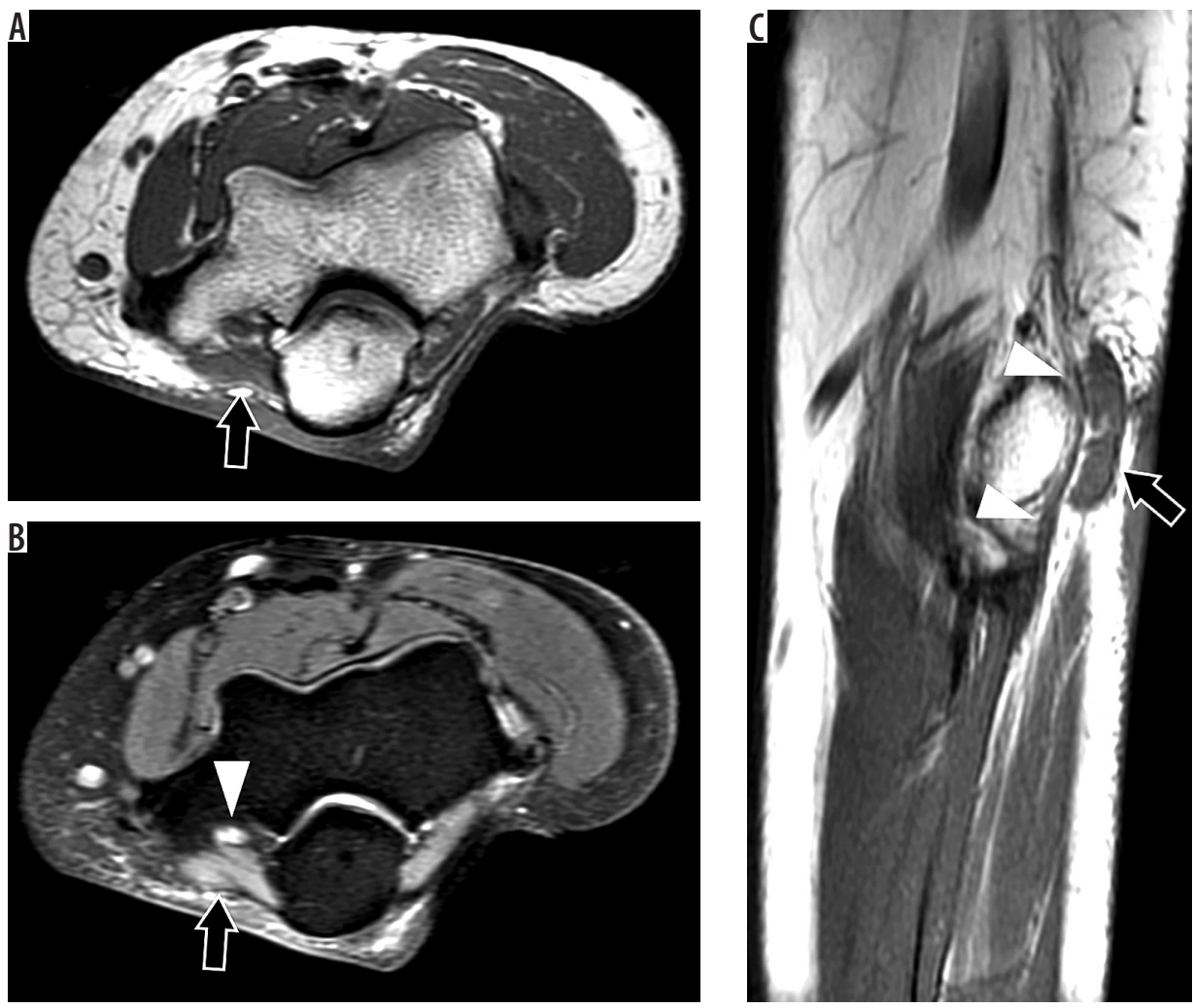

Figure 22. Anconeus epitrochlearis muscle in a 48-year-old female with left elbow pain and limited range of motion. A) Axial T1-weighted and (B) proton density-weighted fat-suppressed magnetic resonance imaging (MRI) shows a large accessory anconeus epitrochlearis muscle at the posteromedial aspect of the elbow (arrows) and mildly hyperintense ulnar nerve (arrowhead). C) Sagittal oblique T1-weighted MRI confirms the large accessory muscle (arrows), which compress the mildly hyperintense ulnar nerve (arrowheads)
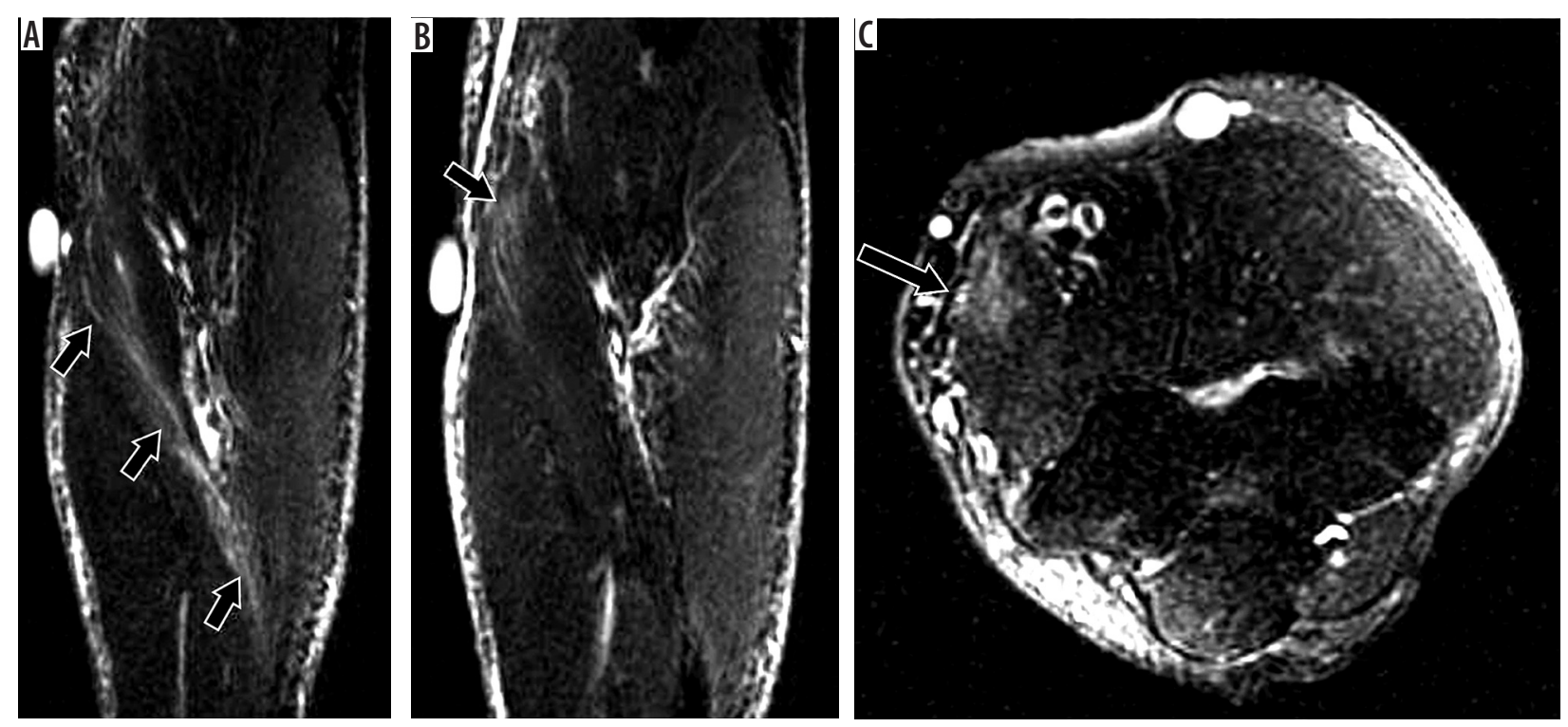

Figure 23. Pronator syndrome. A-B) Coronal oblique and (C) axial proton density-weighted fat-suppressed magnetic resonance imaging of the elbow shows early median nerve denervation changes characterised by mild oedema in the pronator teres muscle (arrows)

Cubital tunnel syndrome is a condition that manifests as medial elbow and forearm pain and paraesthesia, paraesthesia at the medial aspect of the hand, and weakness with loss of coordination of the fingers due to muscle atrophy at the forearm and hand (involvement of the FDP, interossei, medial two lumbricals, and hypothenar muscles).
It may be primary or secondary, caused by nerve dislocation, ganglion cysts, and inflammatory arthropathies [89-92]. The diagnosis starts with clinical examination and electrophysiological studies. MRI plays a significant role in the diagnosis and, as in previously described neuropathies, may show secondary signs of muscle denerva- 

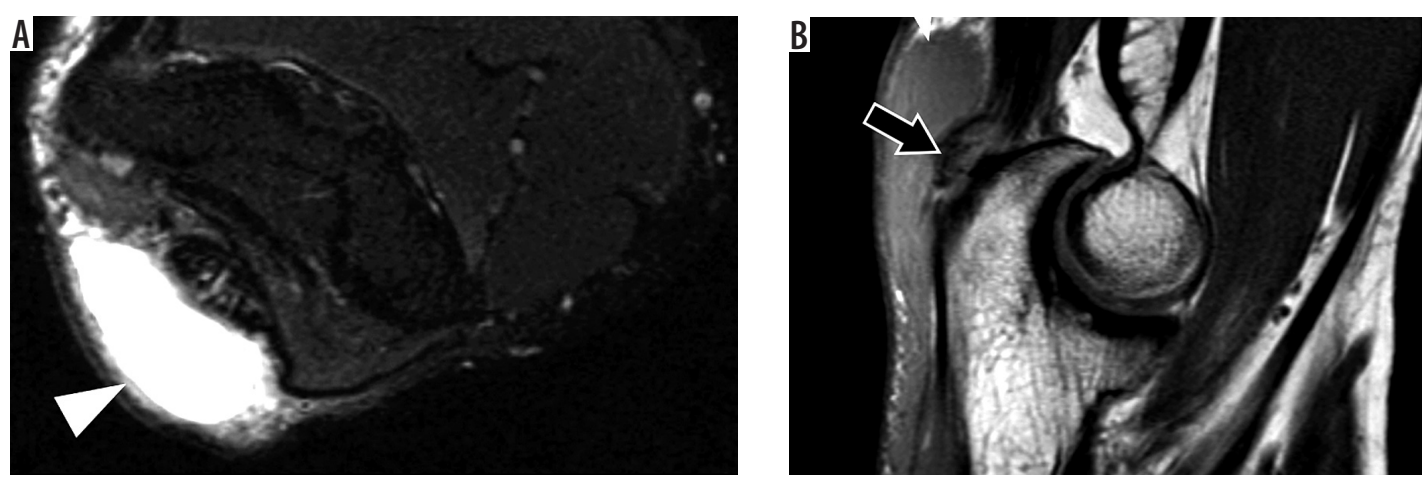

Figure 24. 66-year-old female with olecranon bursitis. A) Axial proton density-weighted fat-suppressed magnetic resonance imaging (MRI) shows fluid distension of the olecranon bursa (arrowhead). B) Sagittal oblique T1-weighted MRI shows the conjoined tendon of the distal triceps inserting onto the fragmented olecranon enthesophyte (arrow) with overlying olecranon bursitis (arrowhead)

tion in the territory of the ulnar nerve (e.g. oedema or atrophy in the flexor muscles of the forearm and hand). MRI can also show local osteophytes, ganglion cysts, or dislocation of the ulnar nerve (Figure 21). The anconeus epitrochlearis, an accessory muscle at the posteromedial aspect of the elbow that runs from the medial epicondyle to the olecranon as previously described, has been implicated as a cause of external compression of the ulnar nerve $[93,94]$ (Figure 22). This anatomical variant, however, was also found in $23 \%$ of asymptomatic patients, thus highlighting the importance of correlation with physical exam findings [95].

The use of MRI for direct assessment of ulnar nerve abnormalities in the setting of cubital tunnel syndrome has been studied in the past. Husarik et al. found that increased signal intensity of the ulnar nerve on fluidsensitive sequences was present in $60 \%$ of asymptomatic patients, suggesting that ulnar neuropathy could be overestimated using this method of evaluation [95]. In a later study by Baumer et al., MR neurography was shown to have high diagnostic accuracy for ulnar neuropathy at the elbow when increased signal intensity on T2-weighted sequences and increased calibre on T1-weighted sequences were present [96]. These findings were later corroborated in a study by Keen $e t$ al., which showed statistically significant differences in nerve size and signal intensity between symptomatic patients and normal volunteers using T1-weighted and fluid-sensitive sequences on MR arthrography, respectively [97]. They also found that the nerve size (with the cut-off of $0.08 \mathrm{~cm}^{2}$ ) showed higher accuracy for the diagnosis of ulnar neuropathy compared to signal alterations, probably because of the high incidence of nerve signal alterations found in normal volunteers. The high false-positive rate in nerve signal alterations was hypothesised as being caused by early changes in nerve signal that could remain clinically silent until the nerve begins to increase in calibre. Another possibility is that the way signal abnormalities were calculated (as focal region of signal alteration), compared to calibre size measurements (average of several points along the course of the nerve), could have played a role. Finally, the inves- tigators suggested that the use of MRI should focus more on the nerve size, or a combination of both, and not only in nerve signal abnormalities [97].

Cubital tunnel syndrome treatment depends on the severity of the neuropathy but starts with conservative measures like arm splinting and rest. Refractory cases may require surgical intervention, which includes decompression, decompression with anterior transposition, or decompression with epicondylectomy $[89,98]$. There are recurrent symptoms in up to $20-35 \%$ of the patients, which makes follow-up imaging of these patients relatively common, requiring some familiarity from the radiologist, with normal and complicated postoperative findings. In medial epicondylectomy and anterior nerve transposition, for example, it is normal to find bone marrow oedema and soft tissue scarring, as well as ulnar nerve thickening. Haematomas, seromas, and soft tissue scarring can cause new nerve compression [99].

\section{Median nerve}

Median neuropathy at the elbow most commonly results from compression between the superficial and deep heads of the pronator teres muscle, hence the name pronator syndrome, but can also be caused by median nerve compression at the bicipital aponeurosis, the origin of flexor muscles, or at the supracondylar process of the humerus [100]. Clinical findings are similar to other neuropathies and include pain and numbness in the area of median nerve distribution, especially during pronation of the forearm. MRI can also show indirect signs of muscle denervation like oedema in the pronator teres or other flexor tendons [34,100] (Figure 23). Anterior interosseous nerve syndrome, also known as Kiloh-Nevin syndrome, is caused by compression of the anterior interosseous nerve (AIN, a motor branch of the median nerve) in the proximal forearm typically distal to the level of entrapment that produces pronator syndrome. Patients with AIN syndrome present with weakness of the thumb and index finger with a disturbance of the pinch mechanism, which may mimic ruptures of the flexor tendons [100]. 

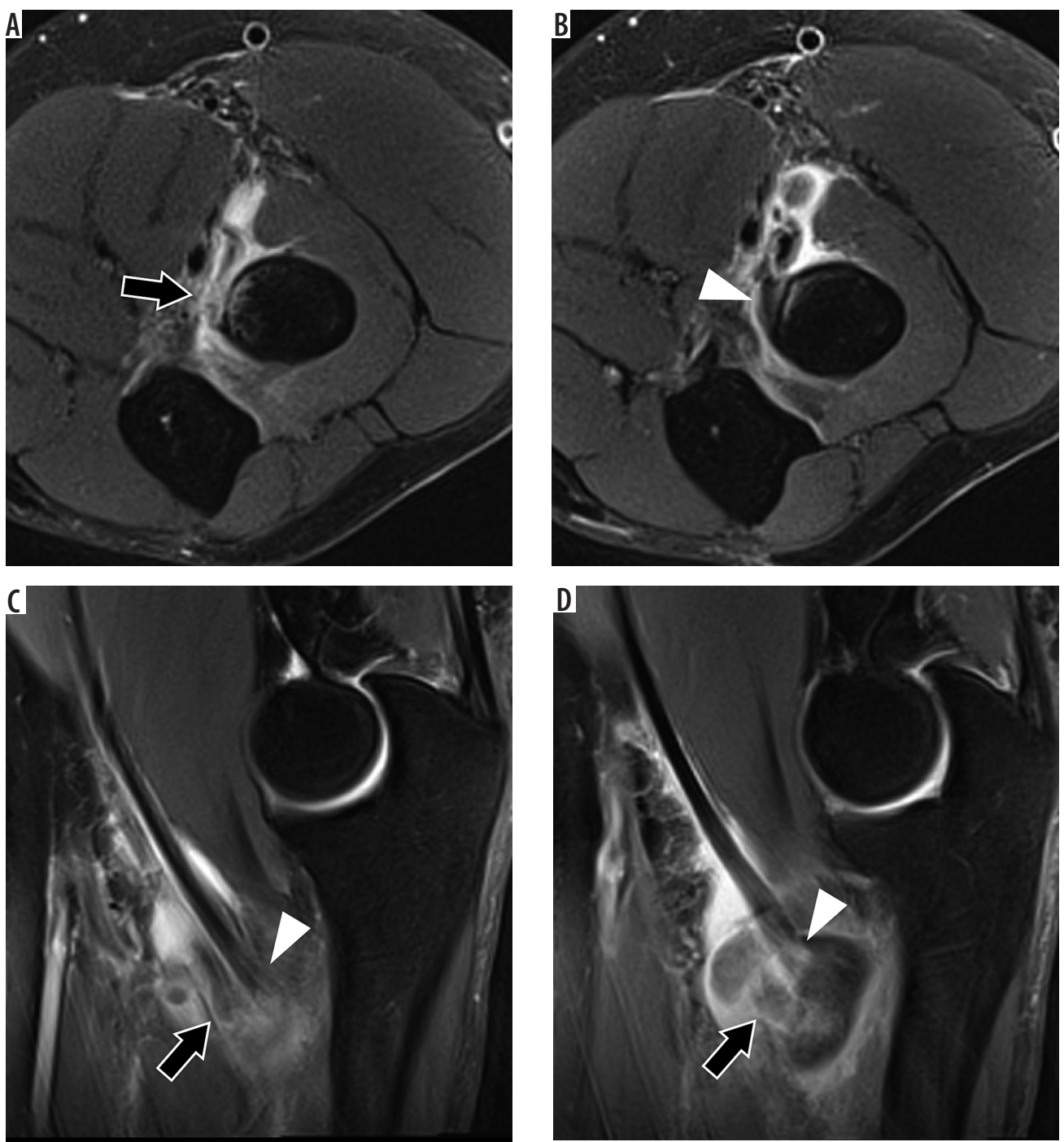

Figure 25. 55-year-old man with left elbow pain after weightlifting injury. A-B axial and C-D sagittal proton density-weighted fat-suppressed magnetic resonance imaging shows a high-grade partial-thickness tear of the short head (arrow) and a low-grade partial-thickness tear of the long head (arrowhead) of the biceps tendon. There is adjacent bone marrow oedema in the radial tuberosity and oedema and haematoma in the bicipitoradial bursa

\section{Bursae and plicae}

Olecranon bursitis may present in the MRI as a collection of fluid in the posterior olecranon subcutaneous region, with thickened walls, with or without septation and gadolinium contrast enhancement. The causes include chronic mechanical friction, inflammatory arthropathies, and infectious conditions [37,101] (Figure 24). Differentiation between septic and aseptic bursitis can be challenging both in the physical exam and imaging because the MRI findings in these conditions show considerable overlap $[102,103]$. Treatment is usually conservative for aseptic aetiologies, with rest, ice, and NSAIDs. In patients with repeated episodes, bursectomy can be considered [104].

Bicipitoradial bursitis is an uncommon condition characterised by bursal fluid distention or synovial proliferation around the distal biceps tendon on MRI. Sometimes it can present as a solid mass with contrast enhancement, often associated with radial tuberosity oedema and erosion, raising concern for a malignant tumour [36,105]. FABS (flexion of the elbow with abduction of the shoulder and supination of the forearm) position can help visualise the typical bursa location involving the biceps tendon, but sometimes imaging is insufficient to establish the aetiology of the bursitis, and tissue sampling and histological analysis are necessary. Biceps tendinopathy and tear, mechanical stress, systemic inflammatory conditions, and infection have been described as potential causes [106,107] (Figure 25). Radial nerve compression and radial tunnel syndrome can also result as an associated complication. Treatment is initially conservative with aspiration and steroid injection; however, surgical excision may be necessary in refractory cases.

Synovial plica syndrome or synovial fringe syndrome is a rare cause of posterolateral elbow pain, usually described as elbow tenderness that increases with movement, associated with mechanical blocking and a snapping sensation. MRI shows long and thick plica at the 
anterior, posterior, or external aspects the radiocapitellar joint. The variation in the plica length and thickness among individuals may cause an overlap between symptomatic and asymptomatic patients, so MRI has to be used as an adjunctive tool in diagnosis and treatment, always accompanied with clinical findings. Lateral epicondylosis must be considered as the main differential diagnosis, given the similar presenting symptoms. Current treatment is arthroscopic plica resection or debridement, although the former has shown better results [39].

\section{Conclusions}

The elbow is a complex joint frequently subjected to trauma and chronic mechanical stress. Understanding its anatomy and biomechanics is the first step towards accurate evaluation and diagnosis of common pathologies using MRI. Diseases that can be observed include bone fractures and joint dislocation, osteochondroses, osteochondritis dissecans, ligament lesions, tendon abnormalities, neuropathies, and bursal conditions. Athletes and children are particularly at-risk populations for chronic overuse syndromes and traumatic injuries. Familiarity with current treatments is also important both to improve communication with the referring physician and to interpret postoperative imaging and surgical complications.

\section{Conflict of interest}

The authors report no conflict of interest.

\section{References}

1. Nordin M, Frankel VH. Basic biomechanics of the musculoskeletal system. 3rd ed. Lippincott Williams \& Wilkins, Philadelphia 2001.

2. Martin S, Sanchez E. Anatomy and biomechanics of the elbow joint. Semin Musculoskelet Radiol 2013; 17: 429-436.

3. O’Driscoll SW, Bell DF, Morrey BF. Posterolateral rotatory instability of the elbow. J Bone Joint Surg Am 1991; 73: 440-446.

4. London JT. Kinematics of the elbow. J Bone Joint Surg Am 1981; 63: 529-535.

5. Prasad A, Robertson DD, Sharma GB, et al. Elbow: the trochleogingylomoid joint. Semin Musculoskelet Radiol 2003; 7: 19-25.

6. Chang $\mathrm{CW}$, Wang $\mathrm{YC}$, Chu $\mathrm{CH}$. Increased carrying angle is a risk factor for nontraumatic ulnar neuropathy at the elbow. Clin Orthop Relat Res 2008; 466: 2190-2195.

7. Giannicola G, Scacchi M, Sedati P, et al. Anatomical variations of the trochlear notch angle: MRI analysis of 78 elbows. Musculoskelet Surg 2016; 100: 89-95.

8. Rosenberg ZS, Beltran J, Cheung YY. Pseudodefect of the capitellum: potential MR imaging pitfall. Radiology 1994; 191: 821-823.

9. Rosenberg ZS, Blutreich SI, Schweitzer ME, et al. MRI features of posterior capitellar impaction injuries. AJR Am J Roentgenol 2008; 190: 435-441.

10. Chung CB, Steinbach LS. MRI of the upper extremity: shoulder, elbow, wrist and hand. Wolters Kluwer Health/Lippincott Williams \& Wilkins, Philadelphia 2010.

11. Schaeffeler C, Waldt S, Woertler K. Traumatic instability of the elbow - anatomy, pathomechanisms and presentation on imaging. Eur Radiol 2013; 23: 2582-2593.

12. Floris S, Olsen BS, Dalstra M, et al. The medial collateral ligament of the elbow joint: anatomy and kinematics. J Shoulder Elbow Surg 1998; 7: 345-351.

13. Yoshida M, Goto H, Takenaga T, et al. Anterior and posterior bands of the anterior bundle in the elbow ulnar collateral ligament: ultrasound anatomy. J Shoulder Elbow Surg 2017; 26: 1803-1809.

14. Timmerman LA, Andrews JR. Histology and arthroscopic anatomy of the ulnar collateral ligament of the elbow. Am J Sports Med 1994; 22: 667-673.
15. Munshi M, Pretterklieber ML, Chung CB, et al. Anterior bundle of ulnar collateral ligament: evaluation of anatomic relationships by using MR imaging, MR arthrography, and gross anatomic and histologic analysis. Radiology 2004; 231: 797-803.

16. Farrow LD, Mahoney AJ, Stefancin JJ, et al. Quantitative analysis of the medial ulnar collateral ligament ulnar footprint and its relationship to the ulnar sublime tubercle. Am J Sports Med 2011; 39: 1936-1941.

17. Magee T. Accuracy of 3-T MR arthrography versus conventional 3-T MRI of elbow tendons and ligaments compared with surgery. AJR Am J Roentgenol 2015; 204: W70-75.

18. Cotten A, Jacobson J, Brossmann J, et al. Collateral ligaments of the elbow: conventional MR imaging and MR arthrography with coronal oblique plane and elbow flexion. Radiology 1997; 204: 806-812.

19. Morrey BF. Reconstruction of the posterior bundle of the medial collateral ligament: a solution for posteromedial olecranon deficiency - a case report. J Shoulder Elbow Surg 2012; 21: e16-19.

20. Hwang JT, Shields MN, Berglund LJ, et al. The role of the posterior bundle of the medial collateral ligament in posteromedial rotatory instability of the elbow. Bone Joint J 2018; 100-B: 1060-1065.

21. Zoner CS, Buck FM, Cardoso FN, et al. Detailed MRI-anatomic study of the lateral epicondyle of the elbow and its tendinous and ligamentous attachments in cadavers. AJR Am J Roentgenol 2010; 195: 629-636

22. Sanal HT, Chen L, Haghighi P, et al. Annular ligament of the elbow: MR arthrography appearance with anatomic and histologic correlation. AJR Am J Roentgenol 2009; 193: W122-126.

23. Mak S, Beltran LS, Bencardino J, et al. MRI of the annular ligament of the elbow: review of anatomic considerations and pathologic findings in patients with posterolateral elbow instability. AJR Am J Roentgenol 2014; 203: 1272-1279.

24. Dirim B, Brouha SS, Pretterklieber ML, et al. terminal bifurcation of the biceps brachii muscle and tendon: anatomic considerations and clinical implications. Am J Roentgenol 2008; 191: W248-W255.

25. Stevens KJ. Magnetic resonance imaging of the elbow. J Magn Reson Imaging 2010; 31: 1036-1053. 
26. Kulshreshtha R, Singh R, Sinha J, et al. Anatomy of the distal biceps brachii tendon and its clinical relevance. Clin Orthop Relat Res 2007; 456: 117-120.

27. Sanal HT, Chen L, Negrao P, et al. Distal attachment of the brachialis muscle: anatomic and MRI study in cadavers. AJR Am J Roentgenol 2009; 192: 468-472.

28. Miyamoto RG, Elser F, Millett PJ. Distal biceps tendon injuries. J Bone Joint Surg Am 2010; 92: 2128-2138.

29. Landa J, Bhandari S, Strauss EJ, et al. The effect of repair of the lacertus fibrosus on distal biceps tendon repairs: a biomechanical, functional, and anatomic study. Am J Sports Med 2009; 37: 120-123.

30. Midtgaard KS, Hallgren HB, Frånlund K, et al. An intact lacertus fibrosus improves strength after reinsertion of the distal biceps tendon. Knee Surg Sports Traumatol Arthrosc 2020; 28: 2279-2284.

31. Negrao JR, Mogami R, Ramirez Ruiz FA, et al. Distal insertional anatomy of the triceps brachii muscle: MRI assessment in cadaveric specimens employing histologic correlation and Play-doh models of the anatomic findings. Skeletal Radiol 2020; 49: 1057-1067.

32. Binaghi D. MR Imaging of the elbow. Magn Reson Imaging Clin N Am 2015; 23: 427-440.

33. Dellon AL. Musculotendinous variations about the medial humeral epicondyle. J Hand Surg Br 1986; 11: 175-181.

34. Andreisek G, Crook DW, Burg D, et al. Peripheral neuropathies of the median, radial, and ulnar nerves: MR imaging features. Radiographics 2006; 26: 1267-1287.

35. Skaf AY, Boutin RD, Dantas RWM, et al. Bicipitoradial bursitis: MR imaging findings in eight patients and anatomic data from contrast material opacification of bursae followed by routine radiography and MR imaging in cadavers. Radiology 1999; 212: 111-116.

36. Yap SH, Griffith JF, Lee RKL. Imaging bicipitoradial bursitis: a pictorial essay. Skeletal Radiol 2019; 48: 5-10

37. Floemer F, Morrison WB, Bongartz G, et al. MRI characteristics of olecranon bursitis. AJR Am J Roentgenol 2004; 183: 29-34

38. Cerezal L, Rodriguez-Sammartino M, Canga A, et al. Elbow synovial fold syndrome. AJR Am J Roentgenol 2013; 201: W88-96.

39. Lee HI, Koh KH, Kim JP, et al. Prominent synovial plicae in radiocapitellar joints as a potential cause of lateral elbow pain: clinicoradiologic correlation. J Shoulder Elbow Surg 2018; 27: 1349-1356.

40. Sampath SC, Sampath SC, Bredella MA. Magnetic resonance imaging of the elbow: a structured approach. Sports Health 2013; 5: 34-49.

41. Chew ML, Giuffrè BM. Disorders of the distal biceps brachii tendon. RadioGraphics 2005; 25: 1227-1237.

42. LiMarzi GM, O’Dell MC, Scherer K, et al. Magnetic resonance arthrography of the wrist and elbow. Magn Reson Imaging Clin N Am 2015; 23: 441-455.

43. Andreisek G, Froehlich JM, Hodler J, et al. Direct MR arthrography at 1.5 and 3.0 T: signal dependence on gadolinium and iodine concentrations - phantom study. Radiology 2008; 247: 706-716.

44. Montgomery DD, Morrison WB, Schweitzer ME, et al. Effects of iodinated contrast and field strength on gadolinium enhancement: implications for direct MR arthrography. J Magn Reson Imaging 2002; 15: 334-343. 45. Kuhn MA, Ross G. Acute elbow dislocations. Orthop Clin North Am 2008; 39: 155-161.

46. Stoneback JW, Owens BD, Sykes J, et al. Incidence of elbow dislocations in the United States population. J Bone Joint Surg Am 2012; 94: 240-245.
47. Hausman MR, Lang P. Examination of the elbow: current concepts. J Hand Surg Am 2014; 39: 2534-2541.

48. Schnetzke M, Schuler S, Hoffend J, et al. Interobserver and intraobserver agreement of ligamentous injuries on conventional MRI after simple elbow dislocation. BMC Musculoskelet Disord 2017; 18: 85.

49. Singer AD, Hanna T, Jose J, et al. A systematic, multimodality approach to emergency elbow imaging. Clin Imaging 2016; 40: 13-22.

50. Dugas JR. Valgus extension overload: diagnosis and treatment. Clin Sports Med 2010; 29: 645-654.

51. Alizai H, Engebretsen L, Jarraya M, et al. Elbow injuries detected on magnetic resonance imaging in athletes participating in the Rio de Janeiro 2016 Summer Olympic Games. J Comput Assist Tomogr 2019; 43: 981-985.

52. Del Grande F, Aro M, Farahani SJ, et al. Three-Tesla MR imaging of the elbow in non-symptomatic professional baseball pitchers. Skeletal Radiol 2015; 44: 115-123.

53. Patel RM, Lynch TS, Amin NH, et al. The thrower's elbow. Orthop Clin North Am 2014; 45: 355-376.

54. Molenaars RJ, van den Bekerom MPJ, Eygendaal D, et al. The pathoanatomy of the anterior bundle of the medial ulnar collateral ligament. J Shoulder Elbow Surg 2019; 28: 1497-1504.

55. Bucknor MD, Stevens KJ, Steinbach LS. Elbow imaging in sport: sports imaging series. Radiology 2016; 279: 12-28.

56. Schwartz ML, al-Zahrani S, Morwessel RM, et al. Ulnar collateral ligament injury in the throwing athlete: evaluation with saline-enhanced MR arthrography. Radiology 1995; 197: 297-299.

57. O’Driscoll SW, Morrey BF, Korinek S, et al. Elbow subluxation and dislocation: a spectrum of instability. Clin Orthop Rel Res 1992; 280: 186-197

58. Jeon IH, Micic ID, Yamamoto N, et al. Osborne-cotterill lesion: an osseous defect of the capitellum associated with instability of the elbow. AJR Am J Roentgenol 2008; 191: 727-729.

59. Erickson SJ, Cox IH, Hyde JS, et al. Effect of tendon orientation on MR imaging signal intensity: a manifestation of the "magic angle" phenomenon. Radiology 1991;181: 389-392.

60. Kwak SH, Lee SJ, Jeong HS, et al. Subtle elbow instability associated with lateral epicondylitis. BMC Musculoskelet Disord 2018; 19: 136.

61. Hayter CL, Adler RS. Injuries of the elbow and the current treatment of tendon disease. AJR Am J Roentgenol 2012; 199: 546-557.

62. Athwal GS, Steinmann SP, Rispoli DM. The distal biceps tendon footprint and relevant clinical anatomy. J Hand Surg 2007; 32: 1225-1229.

63. Eames MH, Bain GI, Fogg QA, et al. Distal biceps tendon anatomy: a cadaveric study. J Bone Joint Surg Am 2007; 89: 1044-1049.

64. Iyer RS, Thapa MM, Khanna PC, et al. Pediatric bone imaging: imaging elbow trauma in children? A review of acute and chronic injuries. Am J Roentgenol 2012; 198: 1053-1068.

65. Laor T, Zbojniewicz AM, Eismann EA, et al. Juvenile osteochondritis dissecans: is it a growth disturbance of the secondary physis of the epiphysis? AJR Am J Roentgenol 2012; 199: 1121-1128.

66. Kijowski R, de Smet AA. MRI findings of osteochondritis dissecans of the capitellum with surgical correlation. Am J Roentgenol 2005; 185: 1453-1459.

67. Laurent LE, Lindstrom BL. Osteochondrosis of the capitulum humeri: panner's disease. Acta Orthop Scand 1956; 26: 111-119. 
68. Kheterpal AB, Bredella MA. Overuse injuries of the elbow. Radiol Clin North Am 2019; 57: 931-942.

69. Jones ADR, Jordan RW. Complex elbow dislocations and the "terrible triad" injury. Open Orthop J 2017; 11: 1394-1404.

70. Sheehan SE, Dyer GS, Sodickson AD, et al. Traumatic elbow injuries: what the orthopedic surgeon wants to know. Radiographics 2013; 33: 869-888.

71. Jackson JD, Steinmann SP. Radial head fractures. Hand Clin 2007; 23: 185-193.

72. Wang ML, Beredjiklian PK. Management of radial head fracture with elbow dislocation. J Hand Surg Am 2015; 40: 813-816.

73. Ring D, Doornberg JN. Fracture of the anteromedial facet of the coronoid process. Surgical technique. J Bone Joint Surg Am 2007; 89: 267-283.

74. Pollock JW, Brownhill J, Ferreira L, et al. The effect of anteromedial facet fractures of the coronoid and lateral collateral ligament injury on elbow stability and kinematics. J Bone Joint Surg Am 2009; 91: 1448-1458.

75. Wiegand L, Bernstein J, Ahn J. Fractures in brief: olecranon fractures. Clin Orthop Relat Res 2012; 470: 3637-3641.

76. Sullivan CW, Desai K. Classifications in brief: Mayo classification of olecranon fractures. Clin Orthop Relat Res 2019; 477: 908-910.

77. Chang EY, Fronek J, Chung CB. Medial supracondylar stress fracture in an adolescent pitcher. Skeletal radiology 2014; 43: 85-88.

78. Smith SR, Patel NK, White AE, et al. Stress fractures of the elbow in the throwing athlete: a systematic review. Orthop J Sports Med 2018; 6: 2325967118799262.

79. Beazley JC, Baraza N, Jordan R, et al. Distal humeral fractures-current concepts. Open Orthop J 2017; 11: 1353-1363.

80. Hochman MG, Zilberfarb JL. Nerves in a pinch: imaging of nerve compression syndromes. Radiol Clin North Am 2004; 42: 221-245.

81. Bencardino JT, Rosenberg ZS. Entrapment neuropathies of the shoulder and elbow in the athlete. Clin Sports Med 2006; 25: 465-487.

82. Naam NH, Nemani S. Radial tunnel syndrome. Orthop Clin North Am 2012; 43: 529-536.

83. Lister GD, Belsole RB, Kleinert HE. The radial tunnel syndrome. J Hand Surg Am 1979; 4: 52-59.

84. Tosun N, Tuncay I, Akpinar F. Entrapment of the sensory branch of the radial nerve (Wartenberg's Syndrome): an unusual cause. Tohoku J Exp Med 2001; 193: 251-254.

85. Rodriguez Miralles J, Natera Cisneros L, Escolà A, et al. Type A ganglion cysts of the radiocapitellar joint may involve compression of the superficial radial nerve. Orthop Traumatol Surg Res 2016; 102: 791-794.

86. Hsiao CM, Wu CC, Wen HC. Compressive radial neuropathy by a synovial cyst during pregnancy: a clinical case report. Medicine (Baltimore) 2018; 97: e13455.

87. Bagga B, Das CJ. MRI of radial cutaneous nerve abscess in recurrent neural leprosy. BMJ Case Rep 2018; 11: e228704.

88. Moradi A, Ebrahimzadeh MH, Jupiter JB. Radial tunnel syndrome, diagnostic and treatment dilemma. Arch Bone J Surg 2015; 3: 156-162.

89. Assmus H, Antoniadis G, Bischoff C, et al. Cubital tunnel syndrome - a review and management guidelines. Cent Eur Neurosurg 2011; 72: 90-98.
90. Komatsu M, Uchiyama S, Kimura T, et al. Recurrent cubital tunnel syndrome caused by ganglion: a report of nine cases. J Hand Surg Asian Pac 2018; 23: 210-216.

91. Alp NB, Akdağ G. Epineural ganglion causing cubital tunnel syndrome: a case report. Jt Dis Relat Surg 2020; 31: 154-158.

92. Andrews K, Rowland A, Pranjal A, et al. Cubital tunnel syndrome: anatomy, clinical presentation, and management. J Orthop 2018; 15: 832-836.

93. Dahners LE, Wood FM. Anconeus epitrochlearis, a rare cause of cubital tunnel syndrome: a case report. J Hand Surg Am 1984; 9: 579-580.

94. Maslow JI, Johnson DJ, Block JJ, et al. Prevalence and clinical manifestations of the anconeus epitrochlearis and cubital tunnel syndrome. Hand (NY) 2020; 15: 69-74.

95. Husarik DB, Saupe N, Pfirrmann CWA, et al. Elbow nerves: MR findings in 60 asymptomatic subjects - normal anatomy, variants, and pitfalls. Radiology 2009; 252: 148-156.

96. Bäumer P, Dombert T, Staub F, et al. Ulnar neuropathy at the elbow: MR neurography - nerve T2 signal increase and caliber. Radiology 2011; 260: 199-206.

97. Keen NN, Chin CT, Engstrom JW, et al. Diagnosing ulnar neuropathy at the elbow using magnetic resonance neurography. Skeletal Radiol 2012; 41: 401-407.

98. Staples JR, Calfee R. Cubital tunnel syndrome: current concepts. J Am Acad Orthop Surg 2017; 25: e215-e224.

99. Rhodes NG, Howe BM, Frick MA, et al. MR imaging of the postsurgical cubital tunnel: an imaging review of the cubital tunnel, cubital tunnel syndrome, and associated surgical techniques. Skeletal Radiol 2019; 48: 1541-1554.

100. Bordalo-Rodrigues M, Rosenberg ZS. MR imaging of entrapment neuropathies at the elbow. Magn Reson Imaging Clin N Am 2004; 12: 247-263.

101. Schweitzer M, Morrison WB. Arthropathies and inflammatory conditions of the elbow. Magn Reson Imaging Clin N Am 1997; 5: 603-617.

102. Floemer F, Morrison WB, Bongartz G, et al. MRI characteristics of olecranon bursitis. Am J Roentgenol 2004; 183: 29-34.

103. Reilly D, Kamineni S. Olecranon bursitis. J Shoulder Elbow Surg 2016; 25: 158-167.

104. Pangia J, Rizvi TJ. Olecranon Bursitis. StatPearls Publishing, Treasure Island 2020.

105. Yee YC, Zhang J, Singbal SB. Bicipitoradial bursitis: a diagnostic dilemma. Am J Phys Med Rehabil 2019; 98: e102-e103.

106. Singh AP, Chadha M, Singh AP, et al. Isolated tuberculous biceps tenosynovitis bicipitoradial bursitis: a case report. J Shoulder Elbow Surg 2009; 18: e30-33.

107. Tsz-Lung C, Tun-Hing L. Bicipitoradial bursitis: a review of clinical presentation and treatment. J Orthop Trauma Rehabil 2014; 18: 7-11. 